\title{
IRREDUCIBLE REPRESENTATIONS OF ODD DEGREE
}

\author{
GABRIEL NAVARRO AND PHAM HUU TIEP
}

\begin{abstract}
McKay's original observation on characters of odd degrees of finite groups is reduced to almost simple groups.
\end{abstract}

\section{INTRODUCTION}

In 1971 John McKay made an observation that changed the course of the Representation Theory of Finite Groups: "In this note we observe that the number $m_{2}(G)$ of inequivalent irreducible complex representations of odd degree of a finite group $G$ is a power of two for many groups $G^{\prime \prime}[\mathrm{M}]$.

What McKay was noticing was a particular (but fundamental) case of what later has become known as the McKay Conjecture: if $G$ is a finite group, $p$ is any prime and $P \in \operatorname{Syl}_{p}(G)$, then

$$
m_{p}(G)=m_{p}\left(\mathbf{N}_{G}(P)\right),
$$

where now $m_{p}(G)$ is the number of inequivalent irreducible complex representations of degree not divisible by $p$ of a finite group $G$. If true, this is an astonishing fact, since global information of a finite group $G$ is going to be encoded in a local small subgroup of $G$, the Sylow normalizer $\mathbf{N}_{G}(P)$.

In the case where $\mathbf{N}_{G}(P)=P$, one has that $m_{p}\left(\mathbf{N}_{G}(P)\right)$ equals the order $\left|P / P^{\prime}\right|$ of the largest abelian quotient of $P$, since the degrees of the irreducible representations of a finite group divide the order of the group. Hence, what McKay was observing was that $m_{2}(G)$ equals the 2-power $\left|P / P^{\prime}\right|$ in the many finite groups having selfnormalizing Sylow 2-subgroups.

In this paper, we prove that in order to prove McKay's original observation, it is enough to check it for certain almost simple groups.

2010 Mathematics Subject Classification. Primary 20C15; Secondary 20C33.

Key words and phrases. McKay conjecture.

The research of the first author is supported by the Prometeo/Generalitat Valenciana, Proyectos MTM2013-40464-P. The second author gratefully acknowledges the support of the NSF (grant DMS1201374) and the Simons Foundation Fellowship 305247.

Part of the paper was written while the second author visited the Department of Mathematics, Harvard University. It is a pleasure to thank Harvard University for generous hospitality and stimulating environment. 
Theorem A. Let $G$ be a finite group with a self-normalizing Sylow 2-subgroup $P$. Suppose that $m_{2}(H)=\left|Q / Q^{\prime}\right|$ whenever $H$ is an almost simple group involved in $G$ with a self-normalizing Sylow 2-subgroup $Q$ and $H=\operatorname{soc}(H) Q$. Then $m_{2}(G)=$ $\left|P / P^{\prime}\right|$.

(Recall that a finite group $H$ is almost simple if $S \triangleleft H \leq \operatorname{Aut}(S)$, where $S$ is a non-abelian simple group. In particular, $S$ is the unique non-trivial normal subgroup of $H$ and therefore the $\operatorname{socle} \operatorname{soc}(H)$ is just $S$. Also, $H$ is involved in a finite group $G$ if there exist subgroups $Y \triangleleft X \leq G$ such that $X / Y \cong H$.)

Our proof of Theorem A uses the Classification of Finite Simple Groups. It is also independent of the ongoing plan proposed in [IMN] (later refined in [S3]) to prove the McKay conjecture: a finite group $G$ will satisfy the McKay Conjecture if every non-abelian simple group $S$ involved in $G$ satisfies the inductive McKay condition. Essentially, $S$ satisfies the inductive McKay condition if there is a bijection between the irreducible characters of $p^{\prime}$-degree of the universal covering group $\hat{S}$ of $S$, and those of its $p$-Sylow normalizer $\mathbf{N}_{\hat{S}}(Q)$, where $Q \in \operatorname{Syl}_{p}(\hat{S})$, that commutes with the action of the automorphisms in $\operatorname{Aut}(\hat{S})$ that stabilize $Q$, respects central characters, and satisfies certain delicate cohomology equalities between character correspondents.

In the statement of our Theorem A there are no cohomology conditions nor covering groups, but a pure reduction of the problem to almost simple groups. This is only possible because, using the self-normalizing condition, we are able to prove a perhaps surprising and deep extension theorem of characters (Theorem 3.3 below) that eliminates cohomology, and that constitutes, we believe, a step further in the plan to check that every finite simple group satisfies the inductive McKay condition. (We note that Theorem 3.3 does not hold without the self-normalizing 2-Sylow condition and that it does not follow from other extendibility results. See Remark 4.15.)

So what is left in order to have a full proof of McKay's original observation? Assume that $S$ is a non-abelian simple group and that $S \leq H \leq \operatorname{Aut}(S)$ is such that $H / S$ is a 2-group and $H$ has a self-normalizing Sylow 2-subgroup $Q$. What is needed is to prove the existence of a bijection between $\operatorname{Irr}_{2^{\prime}}(S)$ and $\operatorname{Irr}_{2^{\prime}}\left(\mathbf{N}_{S}(R)\right)$, where $R=Q \cap S$, that commutes with $Q$-action. If $H=S$, this is a consequence of work by G. Malle and B. Späth [M1, S1, S2]. For $H>S$, unless another argument is found, we need to understand how $Q$ acts on the odd-degree irreducible characters of $S$. How $\operatorname{Out}(S)$ acts on $\operatorname{Irr}(S)$ for a finite simple group of Lie type $S$ is a fundamental problem, which is yet unsolved and currently studied by several mathematicians (including $\mathrm{M}$. Cabanes, G. Lusztig, G. Malle, B. Späth, and others).

In the final section of this paper we offer another application of the extension Theorem 3.3, by proving a reduction to almost simple groups of a well-known conjecture on groups with self-normalizing Sylow 2-subgroups. 


\section{Extending Characters}

First, we recall the "modern notation" for $m_{p}(G)$ which is

$$
m_{p}(G)=\left|\operatorname{Irr}_{p^{\prime}}(G)\right|
$$

where

$$
\operatorname{Irr}_{p^{\prime}}(G)=\{\chi \in \operatorname{Irr}(G) \mid p \nmid \chi(1)\}
$$

and $\operatorname{Irr}(G)$ is the set of the irreducible complex characters of the finite group $G$. In general our notation for characters follows [I2].

We start by proving a new extension theorem of characters which uses Galois conjugation. Let $\sigma \in \operatorname{Gal}(\overline{\mathbb{Q}} / \mathbb{Q})$ be the unique Galois automorphism such that $\sigma$ fixes the 2-power roots of unity and squares the odd roots of unity. This is an automorphism which is related to the McKay Conjecture by work in [N2].

Theorem 2.1. Suppose that $N \triangleleft G, G / N$ has odd order. Let $\theta \in \operatorname{Irr}(N)$ be $G$ invariant, and assume that $\theta^{\sigma}=\theta$. Then $\theta$ extends to $G$, and has a unique extension $\hat{\theta} \in \operatorname{Irr}(G)$ such that

$$
(\hat{\theta})^{\sigma}=\hat{\theta}
$$

Proof. First we prove that if $\theta$ extends, then $\theta$ has a unique extension $\hat{\theta} \in \operatorname{Irr}(G)$ such that $(\hat{\theta})^{\sigma}=\hat{\theta}$. Let $\psi \in \operatorname{Irr}(G)$ be such that $\psi_{N}=\theta$. Now, $\psi^{\sigma}=\lambda \psi$ for some linear $\lambda \in \operatorname{Irr}(G / N)$ by Gallagher's theorem [I2, Corollary (6.17)]. Let $\chi=\bar{\lambda} \psi$. Then $\chi$ extends $\theta$ and

$$
\chi^{\sigma}=(\bar{\lambda} \psi)^{\sigma}=\lambda^{-2} \psi^{\sigma}=\lambda^{-2} \lambda \psi=\bar{\lambda} \psi=\chi .
$$

Suppose now that $\tau_{N}=\theta$ with $\tau^{\sigma}=\tau \in \operatorname{Irr}(G)$. Then $\tau=\mu \chi$ for some linear $\mu \in \operatorname{Irr}(G / N)$. Now

$$
\mu \chi=\tau=\tau^{\sigma}=\mu^{\sigma} \chi^{\sigma}=\mu^{\sigma} \chi=\mu^{2} \chi
$$

and therefore $\mu=\mu^{2}$, by Gallagher's theorem. Hence $\mu=1$, and we have proved what we claimed.

Therefore, it suffices to show that $\theta$ extends to $G$. We argue by induction on $|G: N|$. Let $M / N$ be a maximal normal subgroup of $G / N$. Hence $|G: M|=q$, an odd prime. By induction, $\theta$ has a unique extension $\mu \in \operatorname{Irr}(M)$ such that $\mu^{\sigma}=\mu$. By uniqueness, $\mu$ is $G$-invariant, because $\theta$ is $G$-invariant. Now, $\mu$ extends to $G$ by [I2, Corollary (11.22)], and hence $\theta$ extends to $G$.

Corollary 2.2. Suppose that $N \triangleleft G$. Let $\chi \in \operatorname{Irr}_{2^{\prime}}(G)$, and let $\theta \in \operatorname{Irr}(N)$ be under $\chi$. Let $T=I_{G}(\theta)$ be the stabilizer of $\theta$ in $G$. If $\theta^{\sigma}=\theta$, then $\theta$ extends to $T$.

Proof. Let $\psi \in \operatorname{Irr}(T)$ be the Clifford correspondent of $\chi$ over $\theta$. Then $\psi$ has odd degree, and therefore, we may assume that $\theta$ is $G$-invariant. By [I2, Corollary (11.31)], it suffices to show that $\theta$ extends to $Q$ for every $Q / N \in \operatorname{Syl}_{q}(G / N), q$ any prime. If $q=2$, then $\chi_{Q}$ has some odd degree irreducible constituent $\gamma \in \operatorname{Irr}_{p^{\prime}}(Q)$. Now, 
$\gamma_{N}=\theta$, by [I2, Corollary (11.29)]. If $q$ is odd, then $\theta$ extends to $Q$ by Theorem 2.1 .

The following extension theorem is now a consequence of the McKay-Galois conjecture (proposed by the first author in [N2]). However, this version of the McKay conjecture seems to be very deep and only a few cases have been verified. So, in this form, it can only occasionally be applied. We will remove the dependence of Corollary 2.3 on the McKay-Galois conjecture in Theorem 3.3.

Corollary 2.3. Set $p=2$. Suppose that $N \triangleleft G, \chi \in \operatorname{Irr}_{p^{\prime}}(G)$, and let $\theta \in \operatorname{Irr}(N)$ be under $\chi$. Suppose that $G$ has self-normalizing Sylow 2-subgroups. If the McKayGalois conjecture holds for finite groups with self-normalizing Sylow 2-subgroups, then $\theta$ extends to its stabilizer $T=I_{G}(\theta)$.

Proof. Let $\psi \in \operatorname{Irr}(T)$ be the Clifford correspondent of $\chi$ over $\theta$. Since $\chi$ has $p^{\prime}$-degree, it follows that $T$ contains some $P \in \operatorname{Syl}_{p}(G)$. Since $\psi \in \operatorname{Irr}_{p^{\prime}}(G)$, it follows that some $\tau \in \operatorname{Irr}_{p^{\prime}}(N P)$ under $\psi$ has $p^{\prime}$-degree. We also have that $N P$ has a self-normalizing Sylow 2-subgroup. Therefore, by the McKay-Galois conjecture [N2, Theorem 5.2], we have that $\tau \in \operatorname{Irr}_{p^{\prime}}(N P)$ is $\sigma$-invariant. Since $\tau_{N}=\theta$, then we conclude that $\theta$ is $\sigma$-invariant, and Corollary 2.2 applies.

The following extension lemma is elementary.

Lemma 2.4. Suppose that $N \triangleleft G$ is a p-group. Let $\chi \in \operatorname{Irr}_{p^{\prime}}(G)$, and let $\theta \in \operatorname{Irr}(N)$ be under $\chi$. Then $\theta$ extends to $I_{G}(\theta)$.

Proof. Let $T=I_{G}(\theta)$, and let $\psi \in \operatorname{Irr}(T)$ be the Clifford correspondent of $\chi$ over $\theta$, which has $p^{\prime}$-degree. Hence, we may assume that $T=G$. Let $P \in \operatorname{Syl}_{p}(G)$, which contains $N$. Then $\chi_{P}$ has some $p^{\prime}$-degree irreducible constituent $\mu$ which necessarily lies over $\theta$ and is linear. Thus $\theta$ extends to $P$. But $\theta$ extends to $N Q$ for every $Q \in \operatorname{Syl}_{q}(G)$ with $q \neq p$, by [I2, Corollary (6.28)]. Thus $\theta$ extends to $G$.

If a group $A$ acts by automorphisms on $G$, we will use the notation $\operatorname{Irr}_{A}(G)$ to denote the set of characters in $\operatorname{Irr}(G)$ that are fixed by $A$, and $\operatorname{Irr}_{A, p^{\prime}}(G)$ to denote those $A$-invariant that are of $p^{\prime}$-degree. The following is well-known.

Theorem 2.5. Suppose that $G$ is a finite group, $K=\mathbf{O}^{p}(G)$, and $P \in \operatorname{Syl}_{p}(G)$. Then every $P$-invariant irreducible character of $K$ of $p^{\prime}$-degree extends to $G$. In particular,

$$
\left|\operatorname{Irr}_{p^{\prime}}(G)\right|=\left|\operatorname{Irr}_{P, p^{\prime}}(K)\right| \cdot\left|P:(P \cap K) P^{\prime}\right| .
$$

Proof. If $\chi \in \operatorname{Irr}_{p^{\prime}}(G)$, then $\chi_{K} \in \operatorname{Irr}(K)$ by [I2, Corollary (11.29)]. Now the theorem easily follows from [I2, Corollary (6.28)] and Gallagher's theorem.

We also need a downstairs version of the previous theorem. 
Theorem 2.6. Suppose that $G$ is a finite group, $K=\mathrm{O}^{p}(G)$, and let $P \in \operatorname{Syl}_{p}(G)$. Let $P \leq V \leq G$ and $U=V \cap K$. If $\theta \in \operatorname{Irr}(U)$ has $p^{\prime}$-degree and is $P$-invariant, then $\theta$ extends to $V$.

Proof. We have that

$$
\theta^{K}=\sum_{\substack{\chi \in \operatorname{Irr}_{P}(K) \\\left[\theta^{K}, \chi\right] \chi(1) \equiv 0 \bmod p}}\left[\theta^{K}, \chi\right] \chi+\sum_{\substack{\chi \in \operatorname{Irr}_{P}(K) \\\left[\theta^{K}, \chi\right] \chi(1) \equiv 0 \bmod p}}\left[\theta^{K}, \chi\right] \chi+\sum_{\chi \in \operatorname{Irr}(K) \backslash \operatorname{Irr}_{P}(K)}\left[\theta^{K}, \chi\right] \chi .
$$

Note that the characters in $\operatorname{Irr}(K) \backslash \operatorname{Irr}_{P}(K)$ lie in $P$-orbits of nontrivial $p$-power size and occur with the same multiplicity in the $P$-invariant character $\theta^{K}$. Since $\theta^{K}$ has $p^{\prime}$-degree, we deduce that there exists $\chi \in \operatorname{Irr}(K)$ of $p^{\prime}$-degree, $P$-invariant, such that $\left[\chi, \theta^{K}\right]=\left[\chi_{U}, \theta\right]$ is not divisible by $p$. Now, $\chi$ extends to $G$ by [I2, Corollary (6.28)]. Let $\psi \in \operatorname{Irr}(G)$ be an extension of $\chi$. Then $\left[\psi_{U}, \theta\right]=\left[\chi_{U}, \theta\right]$ is not divisible by $p$. Now,

$$
\left[\psi_{U}, \theta\right]=\sum_{\tau \in \operatorname{Irr}(V)}\left[\psi_{V}, \tau\right]\left[\tau_{U}, \theta\right]
$$

and we deduce that there exists $\tau \in \operatorname{Irr}(V)$ such that $\left[\tau_{U}, \theta\right]$ is not divisible by $p$. Since $\theta$ is $P$-invariant, $\left[\tau_{V}, \theta\right]=\tau(1) / \theta(1)$ divides $|V: U|$ which is a $p$-power. Hence, $\tau_{U}=\theta$, as desired.

Corollary 2.7. Suppose that $K=\mathrm{O}^{p}(G)$ and let $P \in \operatorname{Syl}_{p}(G)$. Let $P \leq V \leq G$ and $U=V \cap K$. Then $V^{\prime} \cap U=[U, V]$.

Proof. We have that $U^{\prime} \leq[U, V] \leq V^{\prime} \cap U$. Let $\lambda \in \operatorname{Irr}(U /[U, V])$. Then $\lambda$ is linear and $V$-invariant. In particular, it is $P$-invariant. Hence, it has an extension $\hat{\lambda} \in \operatorname{Irr}(V)$ by Theorem 2.6. Now, $V^{\prime} \leq \operatorname{Ker}(\hat{\lambda})$ and $V^{\prime} \cap U \leq \operatorname{Ker}(\lambda)$. Since this is true for all $\lambda \in \operatorname{Irr}(U /[U, V])$, we have that $V^{\prime} \cap U \leq[U, V]$.

Finally, we will need an extension theorem from minimal normal subgroups under certain fairly usual circumstances.

Lemma 2.8. Suppose that $N \triangleleft G$, and that $N=S_{1} \times \cdots \times S_{t}$ is the direct product of the set of subgroups $\Omega=\left\{S_{1}, \ldots, S_{t}\right\}$ of $N$ which are transitively permuted by $G$ by conjugation. Write $S=S_{1}$ and view $S / \mathbf{Z}(S) \triangleleft A=\operatorname{Aut}(S)$. Let $\theta=\theta_{1} \times \cdots \times \theta_{t} \in$ $\operatorname{Irr}(N)$ be $G$-invariant, where $\theta_{i} \in \operatorname{Irr}\left(S_{i}\right)$ and $\theta_{1} \in \operatorname{Irr}(S / \mathbf{Z}(S))$. If $\theta_{1}$ extends to $I_{A}\left(\theta_{1}\right)$, then $\theta$ extends to $G$.

Proof. Write $H=\mathbf{N}_{G}(S), S_{i}=S^{x_{i}}$, where $G=\bigcup_{j=1}^{t} H x_{j}$ is a disjoint union. We claim that $\theta_{i}=\left(\theta_{1}\right)^{x_{i}}$. If $s \in S$, then we have that $\theta\left(s^{x_{i}}\right)=\prod_{j \neq i} \theta_{j}(1) \theta_{i}\left(s^{x_{i}}\right)$. Also, using that $\theta$ is $G$-invariant, we have that $\theta\left(s^{x_{i}}\right)=\theta(s)=\prod_{j>1} \theta_{j}(1) \theta_{1}(s)$. Thus $\theta^{x_{i}^{-1}}$ and $\theta_{1}$ are multiple of each other, and therefore they coincide by irreducibility. The claim follows. Now, we notice that $\theta_{1}$ is $H$-invariant because $\theta$ is $G$-invariant. Let 
$C=\mathbf{C}_{G}(S)$. Now, view $S / \mathbf{Z}(S) \cong S C / C \leq H / C \leq A$ and $\theta_{1} \in \operatorname{Irr}(S C / C)$. Since $\theta_{1}$ extends to $I_{A}\left(\theta_{1}\right)$, we have that $\theta_{1}$ extends to $I_{H}\left(\theta_{1}\right)=H$. Let $\psi \in \operatorname{Irr}(H / C)$ be an extension of $\theta_{1}$. Now, let

$$
\rho=\psi^{\otimes G}
$$

be the tensor induced character. (See Section 4 of [I1] for the definition.) By Lemma (4.1) of [I1], we have that

$$
\rho(n)=\prod_{j=1}^{t} \psi\left(x_{j} n x_{j}^{-1}\right)
$$

for $n \in N$. Write $T=\prod_{j=2}^{t} S^{x_{j}}$, so that $N=S \times T$. Notice that $T \leq C \leq \operatorname{Ker}(\psi)$. If we write $n=s_{1} \cdots s_{t}$ with $s_{i} \in S_{i}$, then we have that

$$
\rho(n)=\prod_{j=1}^{t} \theta_{1}\left(s_{j}^{x_{j}-1}\right)=\prod_{j=1}^{t}\left(\theta_{1}^{x_{j}}\right)\left(s_{j}\right)=\theta(n),
$$

and this completes the proof of the lemma.

\section{Proof of Theorem A}

In this section, we prove Theorem A assuming Theorem 3.1 on simple groups, which will be proved in later sections. From now on we set $p=2$.

Theorem 3.1. Let $S$ be a finite non-abelian simple group, and let $A=\operatorname{Aut}(S)$. View $S \triangleleft A$. Suppose that $\theta \in \operatorname{Irr}_{2^{\prime}}(S)$. Let $I=I_{A}(\theta)$ and let $X / S$ be a Sylow 2-subgroup of $I / S$. Suppose that $X$ has a self-normalizing Sylow 2-subgroup. Then $\theta$ extends to $I_{A}(\theta)$.

The self-normalizing hypothesis is conveniently inherited in a key situation.

Lemma 3.2. Suppose that $N \triangleleft G$, and that $N=S_{1} \times \cdots \times S_{k}$ is the direct product of the set of groups $\Omega=\left\{S_{1}, \ldots, S_{k}\right\}$ which are permuted by $G$ by conjugation. Let $P \in \operatorname{Syl}_{p}(G)$ and assume that $P=\mathbf{N}_{N P}(P)$. Then there exists $S_{i}$ such that $Q=$ $P \cap \mathbf{N}_{G}\left(S_{i}\right) \in \operatorname{Syl}_{p}\left(\mathbf{N}_{G}\left(S_{i}\right)\right)$ and $Q=\mathbf{N}_{S_{i} Q}(Q)$.

Proof. Work by induction on $|G|$. First, note by [NTT, Lemma 2.1(ii)] that if $Y \triangleleft$ $X$, where $Y / X$ is a $p$-group and $P \in \operatorname{Syl}_{p}(X)$, then $\mathbf{N}_{X}(P)=P$ if and only if $\mathbf{C}_{\mathbf{N}_{Y}(R) / R}(P)=1$, where $R=P \cap Y$. Now, by Sylow theory, there is some $g \in G$ such that $\mathbf{N}_{G}\left(S_{1}\right) \cap P^{g} \in \operatorname{Syl}_{p}\left(\mathbf{N}_{G}\left(S_{1}\right)\right)$. Now, $\left(S_{1}\right)^{g^{-1}}=S_{i}$ for some $i$, and therefore $Q=P \cap H \in \operatorname{Syl}_{p}(H)$, where $H=\mathbf{N}_{G}\left(S_{i}\right)$.

Write now $N=M \times K$, where $M$ is the product over the $P$-orbit of $S_{i}$ and $K$ is the product of the rest. Notice that $P$ is self-normalizing in $M P$, because it is in $N P$. Assume that $M P<G$. By induction, there is some $x \in P$ such that $R=\mathbf{N}_{M P}\left(S_{i}^{x}\right) \cap$ $P$ is a Sylow $p$-subgroup of $\mathbf{N}_{M P}\left(S_{i}^{x}\right)$, and $R$ is self-normalizing in $S_{1}^{x} R$. Now, $R^{x^{-1}}$ is self-normalizing in $S_{i} R^{x^{-1}}$ and $R^{x^{-1}}=\mathbf{N}_{M P}\left(S_{i}\right) \cap P=\mathbf{N}_{G}\left(S_{i}\right) \cap M P \cap P=Q$, so 
$Q$ is self-normalizing in $S_{i} Q$, as desired. Hence, we may assume that $M P=G$, and that $P$ transitively permutes the set $\Omega$. In particular, $N P=G$.

Now, write $S=S_{1}$, and $N=S^{x_{1}} \times \cdots \times S^{x_{t}}$, where $x_{i} \in P$ and $x_{1}=1$. Recall that $G=N P$. Write $U=Q \cap N=P \cap N \in \operatorname{Syl}_{p}(N)$. Then $P \cap S=Q \cap S=V$,

$$
U=V^{x_{1}} \times \cdots \times V^{x_{t}} \text { and } \mathbf{N}_{N}(U)=\mathbf{N}_{S}(V)^{x_{1}} \times \cdots \times \mathbf{N}_{S}(V)^{x_{t}} .
$$

By hypothesis, we have that $P$ is self-normalizing in $G=N P$. Hence $\mathbf{C}_{\mathbf{N}_{N}(U) / U}(P)=$ 1.

Suppose finally that $z V \in \mathbf{C}_{\mathbf{N}_{S}(V) / V}(Q)$, where $z \in \mathbf{N}_{S}(V)$. Let $y=\prod_{j=1}^{t} z^{x_{j}} \in$ $\mathbf{N}_{N}(U)$. We show that $[y U, P]=U$. In this case $y \in U=V^{x_{1}} \times \cdots \times V^{x_{t}}$, and therefore $z \in V$, which will prove that $Q$ is self-normalizing in $S Q$. Let $x \in P$. We have that

$$
P=\bigcup_{j=1}^{t} Q x_{j} .
$$

Hence $Q x_{j} x=Q x_{\sigma(j)}$, where $\sigma$ is a permutation of $\{1, \ldots, k\}$. Since $z V$ is fixed by $Q$, we have that

$$
\left(z^{x_{j}} V^{x_{j}}\right)^{x}=(z V)^{x_{j} x}=(z V)^{x_{\sigma(j)}} .
$$

Then

$$
(y U)^{x}=\left(z^{x_{1}} V^{x_{1}} \cdots z^{x_{t}} V^{x_{t}}\right)^{x}=\prod_{j}(z V)^{x_{\sigma}(j)} U=y U
$$

as desired.

The following is the fundamental idea to prove Theorem A. (Recall that its proof uses the Classification of Finite Simple Groups.) The case were $p$ is odd was recently proved in $[\mathrm{NTV}]$, with a totally different type of proof, and inspired us to resolve the case $p=2$ here.

Theorem 3.3. Let $G$ be a finite group. Set $p=2$. Let $P \in \operatorname{Syl}_{p}(G)$, and assume that $P=\mathbf{N}_{G}(P)$. Let $N \triangleleft G, \chi \in \operatorname{Irr}_{p^{\prime}}(G)$ and $\theta \in \operatorname{Irr}(N)$ be under $\chi$. Then $\theta$ extends to $I_{G}(\theta)$.

Proof. Let $(G, N)$ be a counterexample with $|N|+|G|$ as small as possible. Let $T=I_{G}(\theta)$, and let $\psi \in \operatorname{Irr}_{p^{\prime}}(T)$ be the Clifford correspondence of $\chi$ over $\theta$. Now, $T$ has $p^{\prime}$-index, and therefore $T$ contains a Sylow $p$-subgroup of $G$ which is therefore self-normalizing in $T$. By minimality, we may assume that $T=G$ and so

$$
\chi_{N}=e \theta .
$$

Suppose that $M \triangleleft G$, where $M<N$, and write $\chi_{M}=f \sum_{i=1}^{t} \tau_{i}$ for a $G$-orbit $\left\{\tau_{1}, \ldots, \tau_{t}\right\}$ on $\operatorname{Irr}(M)$. Since $p \nmid \chi(1)$, we may assume that $\tau=\tau_{1}$ is $P$-invariant. Set $I=I_{G}(\tau)$, so that $t=[G: I]$. By (3.1) we also have that $\theta_{M}=(f / e) \sum_{i=1}^{t} \tau_{i}$, and so $N$ permutes $\tau_{1}, \ldots, \tau_{t}$ transitively, i.e. $t=[N: I \cap N]$ and

$$
G=I N \text {. }
$$


Let $\rho \in \operatorname{Irr}(I \cap N)$ be the Clifford correspondent of $\theta$ over $\tau$. Since $\theta$ and $\tau$ are both $I$-invariant, $\rho$ is $I$-invariant by uniqueness in the Clifford correspondence.

Next, since $\chi$ lies over $\rho$, it follows that some irreducible constituent $\chi_{1}$ of $\chi_{I}$ lies over $\rho$. Hence $\chi_{1}$ lies over $\tau$ and necessarily $\chi=\left(\chi_{1}\right)^{G}$ by the Clifford correspondence. In particular, $\chi_{1}$ has $p^{\prime}$-degree. Now, $P$ is self-normalizing in $I$, and so by induction hypothesis we have that $\rho$ extends to some $\mu \in \operatorname{Irr}(I)$. Applying (3.2) we obtain

$$
\left(\mu^{G}\right)_{N}=\left(\mu_{I \cap N}\right)^{N}=\rho^{N}=\theta
$$

and so we are done in this case. Hence, we may assume that $N$ is a minimal normal subgroup of $G$.

If $N$ is a $p$-group, then we know that $\theta$ extends by Lemma 2.4. If $N$ is a $p^{\prime}$-group, then $\mathbf{N}_{G}(P)=P$ implies that $\mathbf{C}_{N}(P)=1$, and so $\theta=1_{N}$ by the Glauberman correspondence, whence we are done too.

Therefore we may assume that $N$ is a direct product of isomorphic non-abelian simple groups $\left\{S_{1}, \ldots, S_{t}\right\}$ which are transitively permuted by $G$. By Lemma 3.2, there is some $S_{i}$, say $i=1$, and write $S_{1}=S$, such that if $H=\mathbf{N}_{G}(S)$, then $Q=H \cap P \in \operatorname{Syl}_{p}(H)$ and $Q$ is self-normalizing in $S Q$. Write $\theta=\theta_{1} \times \ldots \times \theta_{t}$, where $\theta_{i}=\left(\theta_{1}\right)^{g_{i}}$ for some $g_{i} \in G$ with $S^{g_{i}}=S_{i}$. Now, we have that $S_{1} \leq N \leq H$. Since $\chi$ has $p^{\prime}$-degree, it follows that some irreducible constituent $\xi \in \operatorname{Irr}(H)$ under $\chi$ has $p^{\prime}$-degree. Since $\chi_{S}$ is a multiple of $\theta_{1}$, it follows that $\xi_{S}$ is a multiple of $\theta_{1}$. In particular, $\theta_{1}$ has odd degree and is $H$-invariant. Let $C=\mathbf{C}_{G}(S)$. Then $\bar{\theta}_{1}=\theta_{1} \times 1_{C}$ is $H$-invariant of odd degree. Now, let $\bar{H}=H / C$ and use the bar convention. View $\bar{\theta}_{1} \in \operatorname{Irr}(S C / C)=\operatorname{Irr}(\bar{S})$, which is $\bar{H}$-invariant. We have that $\bar{Q}$ is a Sylow 2subgroup of $\bar{H}$, and that $\bar{Q}$ is self-normalizing in $\bar{S} \bar{Q}$. Write $A=\operatorname{Aut}(\bar{S})$, so we can view $\bar{S} \triangleleft A$, and $\bar{S} \leq \bar{H} \leq J=I_{A}\left(\bar{\theta}_{1}\right) \leq A$. Now let $Y$ be a Sylow 2-subgroup of $J$ containing $\bar{Q}$. Then $\bar{S} \bar{Q} \cap Y=\bar{Q}$, and it easily follows that $Y$ is self-normalizing in $\bar{S} Y=X$ because $\bar{Q}$ is self-normalizing in $\bar{Q} \bar{S}$. By Theorem 3.1, we conclude that $\bar{\theta}_{1}$ extends to $J$. By Lemma 2.8, we now see that $\theta$ extends to $G$, contradicting the choice of $G$ as a minimal counterexample.

In order to prove our main result, we need a relative version of it which implies Theorem A by setting $N=1$.

Theorem 3.4. Let $G$ be a finite group with $P=\mathbf{N}_{G}(P)$, where $P \in \operatorname{Syl}_{p}(G)$ and $p=2$. Let $N \triangleleft G$ and $\theta \in \operatorname{Irr}_{p^{\prime}}(N)$ that extends to $N P$. Assume that $\operatorname{Irr}_{p^{\prime}}(H)=$ $\left|Q / Q^{\prime}\right|$ whenever $H$ is an almost simple group involved in $G$ with a self-normalizing Sylow 2-subgroup $Q$ and $H=\operatorname{soc}(H) Q$. Then

$$
\left|\operatorname{Irr}_{p^{\prime}}(G \mid \theta)\right|=\left|P: P^{\prime}(P \cap N)\right| \text {. }
$$

Proof. Let $(G, N)$ be a counterexample minimizing $|N|+|G|$. Let $T=I_{G}(\theta)$ which contains $P$; in particular, $P$ is self-normalizing in $T$. Then

$$
\left|\operatorname{Irr}_{p^{\prime}}(G \mid \theta)\right|=\left|\operatorname{Irr}_{p^{\prime}}(T \mid \theta)\right|
$$


by the Clifford correspondence, and we may assume that $T=G$. Since $\theta$ extends to $N P$ and has $p^{\prime}$-degree, $\theta$ lies under some $\chi \in \operatorname{Irr}_{p^{\prime}}(G)$ (because $\theta^{G}$ has $p^{\prime}$-degree). By Theorem 3.3, we have that $\theta$ extends to $G$. Thus $\left|\operatorname{Irr}_{p^{\prime}}(G \mid \theta)\right|=\left|\operatorname{Irr}_{p^{\prime}}(G / N)\right|$ by Gallagher's theorem. If $N>1$, then $P N$ is self-normalizing in $G / N$ and by induction $\left|\operatorname{Irr}_{p^{\prime}}(G / N)\right|=\left|P N / P^{\prime} N\right|=\left|P: P^{\prime}(P \cap N)\right|$, and we are done by induction.

Thus we may assume that $N=1$. Let $L$ be a minimal normal subgroup of $G$. Suppose that $L$ is a $p^{\prime}$-group. Then every $\chi \in \operatorname{Irr}_{p^{\prime}}(G)$ lies over some $P$-invariant $\tau \in \operatorname{Irr}(L)$. Since by hypothesis $\mathbf{C}_{L}(P)=1$, we have that $\tau=1_{L}$, by the Glauberman correspondence. Hence $\operatorname{Irr}_{p^{\prime}}(G / L)=\operatorname{Irr}_{p^{\prime}}(G)$, and we are easily done by induction.

Now let $\Delta$ be the set of $p^{\prime}$-degree irreducible characters of $L$ that extend to $L P$. Then

$$
\operatorname{Irr}_{p^{\prime}}(G)=\bigsqcup_{\mu \in \Delta} \operatorname{Irr}_{p^{\prime}}(G \mid \mu)
$$

is a disjoint union. Indeed, if $\chi \in \operatorname{Irr}_{p^{\prime}}(G)$, then some irreducible constituent $\tau$ of $\chi_{L P}$ has $p^{\prime}$-degree, and $\tau_{L} \in \Delta$. Conversely, suppose that $\chi \in \operatorname{Irr}_{p^{\prime}}(G)$ lies over some $P$-invariant $\mu \in \operatorname{Irr}(L)$. If $\nu \in \operatorname{Irr}(L)$ is any $P$-invariant character lying under $\chi$, then we can write $\nu=\mu^{g}$ for some $g \in G$. Now the $P$-invariance of $\nu$ implies that $g P g^{-1} \in I_{G}(\mu)$. Note that $I_{G}(\mu) \geq P$ and $\mathbf{N}_{G}(P)=P$ by hypothesis. It follows that $g \in I_{G}(\mu)$ and $\nu=\mu$. Thus $\mu$ is unique, showing that the union in (3.3) is disjoint.

Write $Q_{0}=L \cap P$ and $R_{0}=Q_{0} P^{\prime}$. By induction, we have that

$$
\left|\operatorname{Irr}_{p^{\prime}}(G \mid \mu)\right|=\left|P: R_{0}\right|
$$

for all $\mu \in \Delta$. Hence (3.3) implies that

$$
\left|\operatorname{Irr}_{p^{\prime}}(G)\right|=|\Delta|\left|P: R_{0}\right| .
$$

We claim that

$$
|\Delta|\left|P: R_{0}\right|=\left|\operatorname{Irr}_{p^{\prime}}(L P)\right| .
$$

If $\mathbf{O}^{p}(L)=L$, then this follows from Theorem 2.5. If $L$ is a $p$-group, then $\Delta$ is the set of different restrictions to $L$ of the linear characters of $P$. This set has size

$$
|\Delta|=\frac{\left|P: P^{\prime}\right|}{\left|P: P^{\prime} L\right|}=\frac{\left|P: P^{\prime}\right|}{\left|P: R_{0}\right|},
$$

and the claim also follows. Combining (3.4) and (3.5), we conclude that

$$
\left|\operatorname{Irr}_{p^{\prime}}(G)\right|=\left|\operatorname{Irr}_{p^{\prime}}(L P)\right| \text {. }
$$

By induction, we may assume that $L P=G$, and certainly that $G$ is not a $p$-group. Hence we have that $L$ (and every other minimal normal subgroup of $G$ ) is a direct product of $t$ non-abelian simple groups of order divisible by $p$ which are transitively permuted by $P$. 
Next, we show that we may assume that $t=1$. The argument is more delicate than expected. Write $L=S^{x_{1}} \times \cdots \times S^{x_{t}}$, where $G=\bigcup_{j=1}^{t} H x_{j}$ with $x_{1}=1$, and $H=\mathbf{N}_{G}(S)$. Thus $G=H P$ and $P \cap H \in \operatorname{Syl}_{p}(H)$. Write $Q=P \cap H$. We have that $Q$ is self-normalizing in $S Q$ by Lemma 3.2. Let $R=L \cap P=L \cap Q$, and let $R_{1}=R \cap S=P \cap S=Q \cap S$. Notice that

$$
R=R_{1}^{x_{1}} \times \cdots \times R_{1}^{x_{t}} .
$$

Furthermore, we claim that $Q=\mathbf{N}_{P}\left(R_{1}\right)$. Since $R_{1}=P \cap S$ and $Q=\mathbf{N}_{P}(S)$, it follows that $Q \leq \mathbf{N}_{P}\left(R_{1}\right)$. Conversely, suppose that $z \in \mathbf{N}_{P}\left(R_{1}\right)$. Let $1 \neq v \in R_{1}$. Then $v^{z} \in R_{1} \leq S$. On the other hand $v^{z} \in S^{z}=S^{x_{j}}$ for some $j$ and $v^{z} \in S \cap S^{x_{j}}$. Necessarily $S^{x_{j}}=S=S^{z}$ and $z \in \mathbf{N}_{P}(S)=Q$. Now, by [NTT, Lemma 4.1(ii)], we deduce that

$$
\left|\operatorname{Irr}_{p^{\prime}, P}(R)\right|=\left|\operatorname{Irr}_{p^{\prime}, Q}\left(R_{1}\right)\right| .
$$

Now, by applying Corollary 2.7 to the groups $G=L P$ and $S Q$, we have that

$$
P^{\prime} \cap R=[P, R] \text { and } Q^{\prime} \cap R_{1}=\left[Q, R_{1}\right] .
$$

Combining this with the equation (3.6), we obtain that

$$
\left|R: P^{\prime} \cap R\right|=\left|R_{1}: Q^{\prime} \cap R_{1}\right| \text {. }
$$

Now, again by [NTT, Lemma 4.1(ii)] we know that

$$
\left|\operatorname{Irr}_{P, p^{\prime}}(L)\right|=\left|\operatorname{Irr}_{Q, p^{\prime}}(S)\right| \text {. }
$$

By Theorem 2.5, we have that

$$
\left|\operatorname{Irr}_{p^{\prime}}(S Q)\right|=\left|\operatorname{Irr}_{Q, p^{\prime}}(S)\right| \cdot\left|Q: Q^{\prime} R_{1}\right|
$$

and

$$
\left|\operatorname{Irr}_{p^{\prime}}(G)\right|=\left|\operatorname{Irr}_{P, p^{\prime}}(L)\right| \cdot\left|P: P^{\prime} R\right|
$$

Suppose that $t>1$. Then $S Q<G$, and by induction applied to $S Q$, we have that

$$
\left|\operatorname{Irr}_{p^{\prime}}(Q S)\right|=\left|Q / Q^{\prime}\right|
$$

Now, by using the equations (3.10), (3.8), (3.9), (3.11) and (3.7), in this order, we obtain that

$$
\left|\operatorname{Irr}_{p^{\prime}}(G)\right|=\left|P: P^{\prime}\right| \text {. }
$$

Hence we may assume that $t=1, L=S$, and that $G=S P$. Since all minimal normal subgroups of $G$ are non-abelian, we also have that $\mathbf{C}_{G}(S)=1$. In this case, the theorem follows by our hypothesis.

\section{Proof of Theorem 3.1}

The goal of this section is to prove Theorem 3.1. 
4.1. Preliminaries. We begin with a reduction.

Lemma 4.1. To prove Theorem 3.1, it suffices to show that $\theta$ extends to $R$, whenever $R / S \in \operatorname{Syl}_{r}(I / S)$ is non-cyclic, $r>2$ a prime, $\theta$ is non-real, and $(S, r)$ is one of the following:

(i) $S=P S L_{n}^{\epsilon}(q)$ with $q=p^{f}$ for some prime $p, \epsilon= \pm, r \mid f$, and $2<r \mid \operatorname{gcd}(n, q-\epsilon)$;

(ii) $S=E_{6}^{\epsilon}(q)$ with $q=p^{f}$ for some prime $p \neq 3, \epsilon= \pm, r=3 \mid f$, and $r \mid(q-\epsilon)$.

Furthermore, one may assume that $\theta$ is not a unipotent character of $S$.

Proof. By Corollaries (11.31) and (11.22) of [I2], it suffices to prove that, for every prime $r$ such that $R / S \in \operatorname{Syl}_{r}(I / S)$ is non-cyclic, $\theta$ extends to $R$. Now if $r=2$, then we are done by [I2, Corollary (8.16)], as o $(\chi)=1$ and $2 \nmid \chi(1)$. Also, if $\theta$ is real, then $\theta$ extends to $I$ by [NT1, Theorem 2.3].

Note that $\operatorname{Out}(S)$ is a 2-group if $S$ is an alternating or sporadic simple group. It remains to consider the simple groups $S$ of Lie type, defined over $\mathbb{F}_{q}$ where $q=p^{f}$ for some prime $p$. If $S=P \Omega_{8}^{+}(q)$, then all $\theta \in \operatorname{Irr}(S)$ are real by [TZ, Theorem 1.2], and so we may assume $S$ is not of type $D_{4}$. Using the description of $\operatorname{Out}(S)$ as given in [GLS, Theorem 2.5.12], one can check that the condition $R / S$ is non-cyclic implies that $(S, r)$ is as listed in (i) or (ii). Finally, if $\theta$ is unipotent, then the result follows from [M2, Theorem 2.4].

Proposition 4.2. Theorem 3.1 holds if $S$ is a simple group of Lie type in characteristic 2 .

Proof. (i) By Lemma 4.1, we need to handle the pairs $(S, r)$ as listed in (i) or (ii) of the Lemma, with $p=2$. We can find a simple algebraic group $\mathcal{H}$ of adjoint type and a Frobenius endomorphism $F: \mathcal{H} \rightarrow \mathcal{H}$ such that $S=[H, H]$ for $H:=\mathcal{H}^{F}$. For instance, $H=P G L_{n}^{\epsilon}(q)$ in the case (i) of Lemma 4.1. Let $\left(\mathcal{H}^{*}, F^{*}\right)$ be dual to $(\mathcal{H}, F)$ and let $H^{*}:=\left(\mathcal{H}^{*}\right)^{F^{*}}$ be dual to $H$. According to the Lusztig classification of irreducible characters of $H$, cf. [C], each $\chi \in \operatorname{Irr}(H)$ is labeled by the $H^{*}$-conjugacy class $s^{H^{*}}$ of some semisimple element $s \in H^{*}$ and a unipotent character $\psi$ of $\mathbf{C}_{H^{*}}(s)$. As $\mathcal{H}$ has trivial center, $\mathbf{C}_{\mathcal{H}^{*}}(s)$ is connected, and so $\mathbf{C}_{H^{*}}(s)$ is a finite connected reductive group defined over $\mathbb{F}_{q}$.

Assume now that $2 \nmid \chi(1)$. Note that $q=2^{f} \geq 8$ in the cases under consideration. It follows by [M1, Theorem 6.8] that any finite non-abelian simple group of Lie type defined over any extension of $\mathbb{F}_{q}$ has exactly one unipotent character of odd degree, namely the principal character. It follows (e.g. by inspecting the structure of $\mathbf{C}_{H^{*}}(s)$ ) that the same is true for $\mathbf{C}_{H^{*}}(s)$, whence $\chi=\chi_{s}$, the semisimple character labeled by $s^{H^{*}}$.

(ii) Let $\tau$ be the involutory graph automorphism of $S$ (mentioned for instance in the proof of [MT, Proposition 4.7]). Here we consider the case $\theta$ is $\tau$-invariant. As $H \triangleleft A=\operatorname{Aut}(S), \tau$ also acts on $H$ and preserves the set $\operatorname{Irr}(H \mid \theta)$. Note that $J / S$ is cyclic of odd order for $J:=I_{H}(\theta)$, as so is $H / S$. By the Clifford correspondence, 
$|\operatorname{Irr}(H \mid \theta)|=|\operatorname{Irr}(J \mid \theta)|=|J / S|$ is odd. It follows that $\tau$ fixes some $\chi \in \operatorname{Irr}(H \mid \theta)$, and $\chi(1)=|H / J| \cdot \theta(1)$ is odd. By (i), $\chi=\chi_{s}$ for some semisimple element $s \in H^{*}$. Now, by Proposition 4.3 and Lemma 4.6 of [MT],

$$
\chi=\chi^{\tau}=\chi_{\tau(s)}=\chi_{s^{-1}}=\bar{\chi}
$$

i.e. $\chi$ is real. Hence the complex conjugation acts on the $\operatorname{set} \operatorname{Irr}(S \mid \chi)$ (of irreducible characters of $S$ lying under $\chi$ ) which is of odd cardinality. This implies that some character $\theta^{\prime} \in \operatorname{Irr}(S \mid \chi)$ is real. As $\theta$ is $H$-conjugate to $\theta^{\prime}$, we see that $\theta$ is real and so we are done by Lemma 4.1 .

In fact, if $\theta^{a}$ is $\tau$-invariant for some $a \in A$, then $\theta^{a}$ is real as shown above, and so extends to $I_{A}\left(\theta^{a}\right)=I^{a}$ (again by [NT1, Theorem 2.3]), whence $\theta$ extends to $I$ and we are done. From now on we will assume that $\theta$ is not $\tau^{a}$-invariant for any $a \in A$.

In the cases where $H={ }^{2} E_{6}(q)$ ad or $H=P G U_{n}(q)$, we have $\operatorname{Out}(S)=H / S \rtimes C_{2 f}$. If furthermore $X>S$, then $X / S$ contains an $H$-conjugate of the $\operatorname{coset} \tau S$ and so $\theta$ is invariant under some $H$-conjugate of $\tau$, contrary to our assumption. Hence $X=S$. But in this case, $\mathbf{N}_{X}(Q)>Q$ for $Q \in \operatorname{Syl}_{2}(S)$ (indeed, $Q$ is normalized by some torus of order $q+1$ in ${ }^{2} E_{6}(q)_{\mathrm{sc}}$, respectively in $\left.S U_{n}(q)\right)$.

(iii) In the remaining cases, $\operatorname{Out}(S)=H / S \rtimes D$, where $D=C_{2} \times C_{f}$. Let $\sigma$ denote the field automorphism of $S$ coming from the map $x \mapsto x^{2}$ of $\overline{\mathbb{F}}_{2}$.

Consider the case $S=P S L_{n}(q)$ (recall $n \geq r \geq 3$ ) and let $L=S L_{n}(q)=S L(V)$, where $V=\left\langle e_{1}, \ldots, e_{n}\right\rangle_{\mathbb{F}_{q}}$. We can embed $Q \in \operatorname{Syl}_{2}(S)$ in $L$ as the subgroup of all upper unitriangular matrices and have $\mathbf{N}_{L}(Q)=Q T$, where $T$ is the diagonal subgroup. Also, $Q T$ is normalized by $\sigma$ and by $\varphi:=\nu \tau$, where $\nu$ is the conjugation by the element of $L$ that sends $e_{i}$ to $e_{n+1-i}, 1 \leq i \leq n$, and $\tau(g)={ }^{t} g^{-1}$ for all $g \in L$. Note that we can choose $D$ so that $D=\langle\varphi\rangle \times\langle\sigma\rangle$. Replacing $\theta$ by a suitable $H$-conjugate, we may assume that $X / S \leq D$. Now, $\varphi$ acts on $T$ via

$$
\operatorname{diag}\left(t_{1}, t_{2}, \ldots, t_{n-1}, t_{n}\right) \mapsto \operatorname{diag}\left(t_{n}^{-1}, t_{n-1}^{-1}, \ldots, t_{2}^{-1}, t_{1}^{-1}\right) .
$$

Write $f=2^{c} f_{1}$ with $2 \nmid f_{1}$ and consider $\sigma_{1}:=\sigma^{f_{1}}$. Then $\left\langle\varphi, \sigma_{1}\right\rangle$ is the unique Sylow 2-subgroup of $D$, and it acts trivially on the subgroup

$$
T_{1}:=\left\{\operatorname{diag}\left(t, 1, \ldots, 1, t^{-1}\right) \mid t^{2^{f_{1}-1}}=1\right\}
$$

of $T$. As $r \mid f$, we see that $f_{1} \geq r \geq 3$, and so $\left|T_{1}\right| \geq 7$; furthermore, $T_{1} \cap \mathbf{Z}(L)=1$. Embedding $Q$ in a Sylow 2-subgroup $R$ of $Q T \rtimes\left\langle\varphi, \sigma_{1}\right\rangle$, we now see that $R \in \operatorname{Syl}_{2}(Y)$ for $Y:=S \rtimes\left\langle\varphi, \sigma_{1}\right\rangle$ and that $\mathbf{C}_{\mathbf{N}_{S}(Q) / Q}(R) \neq 1$. Hence $R$ is not self-normalizing in $Y$ by [NTT, Lemma 2.1]. As $S \triangleleft X \leq Y, X$ cannot have a self-normalizing Sylow 2-subgroup, a contradiction.

Finally, in the case $S$ is of type $E_{6}$, we can view $S=M / \mathbf{Z}(M)$ for $M:=E_{6}(q)_{\mathrm{sc}}$. We can embed $Q \in \operatorname{Syl}_{2}(S)$ first in $M$ and then in a $D$-invariant maximal parabolic subgroup of type $A_{5}$ with Levi subgroup $S L_{6}(q) \cdot C_{q-1}$. The above arguments applied 
to $S L_{6}(q)$ (which is $D$-invariant) show that $X$ cannot have a self-normalizing Sylow 2-subgroup, a contradiction.

Lemma 4.3. Let $p>2$ be a prime, $q=p^{f}$, and let $\epsilon= \pm$.

(i) Suppose that $n=2 m$ for some natural integer $m$. Then $\left|G L_{n}^{\epsilon}(q)\right| /\left|G L_{m}^{\epsilon}(q)\right|^{2}$ is even for all $m$, and is divisible by 4 if $2 \nmid m>1$.

(ii) Suppose that $n=k+l$ for some odd integers $k, l \geq 1$. Then $\mid G L_{n}(q): G L_{k}(q) \times$ $G L_{l}(q) \mid$ is even.

(iii) Suppose that $n=2^{a}$ for some natural integers $a, m$. Then

$$
2^{2^{a}-1} \mid \frac{\left|G L_{n}^{\epsilon}(q)\right|}{\left|G L_{m}^{\epsilon}(q)\right|^{2^{a}}} .
$$

(iv) Suppose that $n=k m$ for some integers $k \geq 2$ and $m \geq 1$. Then

$$
2 \mid \frac{\left|G L_{n}^{\epsilon}(q)\right|}{\left|G L_{m}^{\epsilon}(q)\right|^{k}} .
$$

Proof. We prove the statements for $\epsilon=+$. The case $\epsilon=-$ is proved in the same way, replacing $q$ by $-q$.

(i) Note that

$N:=\left|G L_{2 m}(q): G L_{m}(q) \times G L_{m}(q)\right|_{p^{\prime}}=\left(q^{m}+1\right) \cdot\left|G L_{2 m-1}(q): G L_{m}(q) \times G L_{m-1}(q)\right|_{p^{\prime}}$ is even. Moreover, if $m=2 k+1 \geq 3$, then

$$
\begin{gathered}
N=\frac{\left(q^{2 k+2}-1\right)\left(q^{2 k+3}-1\right) \ldots\left(q^{4 k+2}-1\right)}{(q-1)\left(q^{2}-1\right) \ldots\left(q^{2 k+1}-1\right)} \\
=\frac{\left(q^{2 k+3}-1\right)\left(q^{2 k+5}-1\right) \ldots\left(q^{4 k+1}-1\right)}{\left(q^{3}-1\right)\left(q^{5}-1\right) \ldots\left(q^{2 k+1}-1\right)} \cdot \frac{\left(q^{2 k+2}-1\right)\left(q^{2 k+4}-1\right) \ldots\left(q^{4 k}-1\right)}{\left(q^{2}-1\right)\left(q^{4}-1\right) \ldots\left(q^{2 k}-1\right)} \cdot \frac{q^{4 k+2}-1}{q-1} .
\end{gathered}
$$

The first factor in the last product has 2-adic valuation 0 . The second factor equals

$$
\left(q^{2 k}+1\right) \cdot\left[G L_{2 k-1}\left(q^{2}\right): G L_{k}\left(q^{2}\right) \times G L_{k-1}\left(q^{2}\right)\right]_{p^{\prime}}
$$

and so is even. The third is also even, and so we are done.

(ii) We have

$$
\left|G L_{n}(q): G L_{k}(q) \times G L_{l}(q)\right|_{p^{\prime}}=\left|G L_{n-1}(q): G L_{k}(q) \times G L_{l-1}(q)\right|_{p^{\prime}} \cdot \frac{q^{n}-1}{q-1} \cdot \frac{q-1}{q^{l}-1} .
$$

Since the last factor has 2 -adic valuation 0 and the middle is even, this product is even.

(iii) We proceed by induction on $a \geq 2$, with the induction base $a=1$ following from (i). Suppose that the statement holds for $n=2^{a} m$. Then

$$
\frac{\left|G L_{2^{a+1} m}(q)\right|}{\left|G L_{m}(q)\right|^{2^{a+1}}}=\frac{\left|G L_{2 n}(q)\right|}{\left|G L_{n}(q)\right|^{2}} \cdot\left(\frac{\left|G L_{2^{a} m}(q)\right|}{\left|G L_{m}(q)\right|^{2^{a}}}\right)^{2}
$$


is divisible by $2^{1+2\left(2^{a}-1\right)}=2^{2^{a+1}-1}$, as desired.

(iv) We proceed by induction on $k \geq 2$, with the induction base again following from (i). For the induction step, note

$$
\frac{\left|G L_{(k+1) m}(q)\right|}{\left|G L_{m}(q)\right|^{k+1}}=\frac{\left|G L_{m(k+1)}(q)\right|}{\left|G L_{k m}(q)\right| \cdot\left|G L_{m}(q)\right|} \cdot \frac{\left|G L_{k m}(q)\right|}{\left|G L_{m}(q)\right|^{k}} .
$$

Lemma 4.4. Let $n, k$ be integers with $0 \leq k \leq n$, and let $q \neq \pm 1$ be an odd integer.

(i) $\left(\begin{array}{l}n \\ k\end{array}\right)$ is odd if and only if

$$
f(q):=\frac{(q-1)\left(q^{2}-1\right) \ldots\left(q^{n}-1\right)}{(q-1)\left(q^{2}-1\right) \ldots\left(q^{k}-1\right) \cdot(q-1)\left(q^{2}-1\right) \ldots\left(q^{n-k}-1\right)}
$$

is odd.

(ii) If $n=2^{a}$ then $2 \nmid\left(\begin{array}{l}n \\ k\end{array}\right)$ precisely when $k=0$, n. If $n=2^{a}+2^{b}$ with $a>b \geq 0$, then $2 \nmid\left(\begin{array}{l}n \\ k\end{array}\right)$ precisely when $k \in\left\{0, n, 2^{a}, 2^{b}\right\}$.

Proof. (i) It is well known that $f(q)$ is a polynomial in $q$ with integer coefficients. It follows that $f(q) \equiv f(-q)(\bmod 2)$. Replacing $q$ by $-q$ if necessary, we may assume that $q \equiv 1(\bmod 4)$. In this case, $\left(q^{j}-1\right)_{2}=(q-1)_{2} \cdot j_{2}$ for $1 \leq j \leq n$, whence $f(q)_{2}=\left(\begin{array}{l}n \\ k\end{array}\right)_{2}$.

(ii) Suppose first that $n=2^{a}$ and $0<k<n$. Then

$$
\left\lfloor n / 2^{a}\right\rfloor-\left\lfloor k / 2^{a}\right\rfloor-\left\lfloor(n-k) / 2^{a}\right\rfloor=1,
$$

whence $2 \mid\left(\begin{array}{l}n \\ k\end{array}\right)$. Next suppose that $n=2^{a}+2^{b}$ and $0<k<n / 2$; in particular, $k<2^{a}$. If moreover $k>2^{b}$, then $n-k<2^{a}$ and so

$$
\left\lfloor n / 2^{a}\right\rfloor-\left\lfloor k / 2^{a}\right\rfloor-\left\lfloor(n-k) / 2^{a}\right\rfloor=1,
$$

implying $2 \mid\left(\begin{array}{l}n \\ k\end{array}\right)$. If $k<2^{b}$, then $2^{a-b}<(n-k) / 2^{b}<2^{a-b}+1$, and so

$$
\left\lfloor n / 2^{b}\right\rfloor-\left\lfloor k / 2^{b}\right\rfloor-\left\lfloor(n-k) / 2^{b}\right\rfloor=1,
$$

again implying $2 \mid\left(\begin{array}{l}n \\ k\end{array}\right)$. If $k=2^{b}$, then one can check that $2 \nmid\left(\begin{array}{l}n \\ k\end{array}\right)$.

4.2. Groups of type $A$. A description of height 0 characters of finite groups of Lie type (in cross characteristic) was obtained in [M2]. However, for our purposes it is more convenient to have another description of these characters in the cases of types $A$ and $E_{6}$.

Lemma 4.5. Let $L=S L_{n}(q)$ and $G=G L_{n}(q)$ with $n \geq 3$ and $q=p^{f}$ for some odd prime $p$. Let $\theta \in \operatorname{Irr}_{2^{\prime}}(L)$ be non-unipotent and let $\chi \in \operatorname{Irr}(G \mid \theta)$. Identify $G^{*}$ with $G$ and let $s \in G$ be a semisimple element such that $\chi$ belongs to the rational Lusztig series $\mathcal{E}(G,(s))$ labeled by $s^{G}$. Then one of the following statements holds for $C:=\mathbf{C}_{G}(s)$. 
(a) $\chi(1)=\theta(1)$ and $C \cong \prod_{i=1}^{m} G L_{k_{i}}(q)$ with $m>1, k_{1}<\ldots<k_{m}$, and $\sum_{i=1}^{m} k_{i}=n$. Moreover, $s$ is $G$-conjugate to

$$
\operatorname{diag}(\underbrace{\alpha_{1}, \ldots, \alpha_{1}}_{k_{1}}, \ldots, \underbrace{\alpha_{m}, \ldots, \alpha_{m}}_{k_{m}})
$$

for some pairwise distinct $\alpha_{1}, \ldots, \alpha_{m} \in \mathbb{F}_{q}^{\times}$.

(b) $\chi(1)=2 \theta(1), C \cong G L_{k}(q)^{2}, n=2 k$, and $2 \mid k$. Moreover, $s$ is $G$-conjugate to

$$
\operatorname{diag}(\underbrace{\alpha, \ldots, \alpha}_{k}, \underbrace{-\alpha, \ldots,-\alpha}_{k})
$$

for some $\alpha \in \mathbb{F}_{q}^{\times}$.

Proof. (i) Note that $[G: C]_{p^{\prime}} \mid \chi(1)$ by Lusztig's parametrization of $\operatorname{Irr}(G)$. Since $G / L$ is cyclic of order $q-1$ and $2 \nmid \theta(1)$, we see that $2(q-1) \nmid \chi(1)$; in particular, $2(q-1) \nmid[G: C]$. It is well known that there are some integers $m \geq 1, k_{i}, a_{i} \geq 1,1 \leq$ $i \leq m$, such that $n=\sum_{i=1}^{m} k_{i} a_{i}$ and $C \cong \prod_{i=1}^{m} G L_{k_{i}}\left(q^{a_{i}}\right)$, where the factor $G L_{k_{i}}\left(q^{a_{i}}\right)$ corresponds to an eigenvalue $\alpha_{i}$ of $s$ of degree $a_{i}$ over $\mathbb{F}_{q}$ and with multiplicity $k_{i}$. Now, if $k_{i} a_{i} \geq 3$ and $a_{i} \geq 2$ for some $i$, then $\left[G L_{k_{i} a_{i}}(q): G L_{k_{i}}\left(q^{a_{i}}\right)\right]_{p^{\prime}}$ is divisible by

$$
\prod_{1 \leq j<k_{i} a_{i}, a_{i} \nmid j}\left(q^{j}-1\right)
$$

which is divisible by $(q-1)^{2}$, a contradiction. Similarly, if $k_{1} a_{1}=k_{2} a_{2}=2$ and $a_{1}=a_{2}=2$, then

$$
\left[G L_{k_{1} a_{1}+k_{2} a_{2}}(q): G L_{k_{1}}\left(q^{a_{1}}\right) \times G L_{k_{2}}\left(q^{a_{2}}\right)\right]_{p^{\prime}}=\left[G L_{4}(q): G L_{1}\left(q^{2}\right) \times G L_{1}\left(q^{2}\right)\right]_{p^{\prime}}
$$

is again divisible $(q-1)^{2}$, a contradiction. So either $a_{i}=1$ for all $i$, or, say, $a_{1}=$ $k_{1} a_{1}=2$ and $a_{2}=\ldots=a_{m}=1$.

(ii) Next, we consider

$$
J:=\{\lambda \in \operatorname{Irr}(G / L) \mid \chi \lambda=\chi\}
$$

as a cyclic group under multiplication, of order dividing $q-1=|G / L|$. By $[\mathrm{KT}$, Lemma 3.2(i)], $\chi(1) / \theta(1)=|J|$. There is a group isomorphism $z \mapsto \lambda_{z}$ between $\mathbf{Z}(G)$ and $\operatorname{Irr}(G / L)$ such that the multiplication by $\lambda_{z}$ sends the rational Lusztig series $\mathcal{E}(G,(s))$ to $\mathcal{E}(G,(s z))$, cf. [L, (7.4.2), (7.5.5)]. As Lusztig series are disjoint, it follows that $s$ and $s z$ are conjugate in $G$ whenever $\lambda_{z} \in J$. to

Suppose that $a_{1}=k_{1} a_{1}=2$ and $a_{2}=\ldots=a_{m}=1$. Then $s$ is $G L_{n}\left(\overline{\mathbb{F}}_{q}\right)$-conjugate

$$
\operatorname{diag}(\alpha_{1}, \alpha_{1}^{q}, \underbrace{\alpha_{2}, \ldots, \alpha_{2}}_{k_{2}}, \ldots, \underbrace{\alpha_{m}, \ldots, \alpha_{m}}_{k_{m}}),
$$

where $\alpha_{1} \in \mathbb{F}_{q^{2}} \backslash \mathbb{F}_{q}$, and $\alpha_{2}, \ldots, \alpha_{m} \in \mathbb{F}_{q}^{\times}$are pairwise distinct. Note that

$$
\left[G L_{k_{1} a_{1}}(q): G L_{k_{1}}\left(q^{a_{1}}\right)\right]_{p^{\prime}}=\left[G L_{2}(q): G L_{1}\left(q^{2}\right)\right]_{p^{\prime}}=q-1
$$


and so $(q-1) \mid[G: C]_{p^{\prime}}$. In particular, if $2 \nmid|J|$, then $\theta(1)=\chi(1) /|J|$ is even, a contradiction. Thus $|J|$ is even, and so $\lambda_{t} \in J$, where $t=-1_{V}$ and $V=\mathbb{F}_{q}^{n}$ denotes the natural $G$-module. This in turn implies that $s$ and $-s$ are $G$-conjugate. As $n>2$, we see that $m \geq 2$ and the multiplication by -1 preserves the multi-set $\operatorname{Spec}(s)$ of eigenvalues of $s$ (counted with multiplicities). Renaming the $\alpha_{i}$ 's if necessary, we may assume that $\alpha_{3}=-\alpha_{2}$ and $k_{3}=k_{2}$. By Lemma 4.3(i),

$$
\left[G L_{k_{2}+k_{3}}(q): G L_{k_{2}}(q) \times G L_{k_{3}}(q)\right]_{p^{\prime}}
$$

is even. Together with $(4.1)$, this implies that $2(q-1) \mid[G: C]_{p^{\prime}}$, a contradiction.

(iii) We have shown that $a_{1}=\ldots=a_{m}=1$. As $\theta$ is non-unipotent, [L, (7.5.5)] implies that $s \notin \mathbf{Z}(G)$, and so $m \geq 2$.

Assume first that $|J|$ is odd. If not all $k_{i}$ are distinct, for instance, $k_{1}=k_{2}$, then

$$
\left[G L_{k_{1}+k_{2}}(q): G L_{k_{1}}(q) \times G L_{k_{2}}(q)\right]_{p^{\prime}}
$$

is even by Lemma $4.3(\mathrm{i})$ and so $\theta(1)=\chi(1) /|J|$ is even, a contradiction. Hence the $k_{i}$ 's are pairwise distinct. If in addition $|J|>1$, then $J \ni \lambda_{z}$ for some $1 \neq z=$ $\beta \cdot 1_{V} \in \mathbf{Z}(G)$, and $s$ and $s z$ are $G$-conjugate. Hence the multiplication by $\beta$ acts on $\operatorname{Spec}(s)$, sending, say, $\alpha_{1}$ to $\alpha_{2}$ and then forcing $k_{1}=k_{2}$, again a contradiction. Thus we have arrived at the conclusion (a).

It remains to consider the case $|J|=2^{a} t$ with $a \geq 1$ and $2 \nmid t \geq 1$. Then $J \ni \lambda_{z}$, where $z=\gamma \cdot 1_{V} \in \mathbf{Z}(G)$ and $\gamma \in \mathbb{F}_{q}^{\times}$has order $2^{a}$. As above, the multiplication by $\gamma$ acts semi-regularly on $\operatorname{Spec}(s)$. Without loss, we may assume that $\left\{\alpha_{1}, \alpha_{2}, \ldots, \alpha_{r}\right\}$ is one orbit for this action, where $r=2^{a}$. In this case, $k_{1}=\ldots=k_{r}$, and

$$
\left[G L_{k_{1}+k_{2}+\ldots+k_{r}}(q): G L_{k_{1}}(q) \times G L_{k_{2}}(q) \times \ldots \times G L_{k_{r}}(q)\right]_{p^{\prime}}
$$

is divisible by $2^{r-1}$ by Lemma 4.3 (iii). If $a>1$ in addition, then $r-1 \geq a+1$, and so $\theta(1)=\chi(1) / 2^{a} t$ is even, a contradiction. It follows that $a=1$ and $\gamma=-1$. Now, if $m>2$, then we may assume that $\left(k_{2}, \alpha_{2}\right)=\left(k_{1},-\alpha_{1}\right),\left(k_{4}, \alpha_{4}\right)=\left(k_{3},-\alpha_{3}\right)$, and Lemma 4.3(i) implies that

$$
\left[G L_{k_{1}+k_{2}+k_{3}+k_{4}}(q): G L_{k_{1}}(q) \times G L_{k_{2}}(q) \times G L_{k_{3}}(q) \times G L_{k_{r}}(q)\right]_{p^{\prime}}
$$

is divisible by 4 , in which case $\theta(1)=\chi(1) / 2 t$ is even. So $m=2$, and $s$ has precisely two distinct eigenvalues $\alpha=\alpha_{1}$ and $\alpha_{2}=-\alpha$, both with multipliticity $k=n / 2>1$. Choosing a generator $\lambda_{\delta \cdot 1_{V}}$ for $J$, where $\delta \in \mathbb{F}_{q}^{\times}$has order $2 t$, we again have that the multiplication by $\delta$ acts on $\operatorname{Spec}(s)$. It follows that $\delta=-1$ and $t=1$. Applying Lemma 4.3(i) again, we see that $2 \mid k$, yielding the conclusion (b).

In what follows, we denote by $\mu_{q+1}$ the group $\left\{\alpha \in \mathbb{F}_{q^{2}}^{\times} \mid \alpha^{q+1}=1\right\}$ (under multiplication). We will also sometimes denote by $\mu_{q-1}$ the multiplicative group $\mathbb{F}_{q}^{\times}$.

Lemma 4.6. Let $L=S U_{n}(q)$ and $G=G U_{n}(q)$ with $n \geq 3$ and $q=p^{f}$ for some odd prime $p$. Let $\theta \in \operatorname{Irr}_{2^{\prime}}(L)$ be non-unipotent and let $\chi \in \operatorname{Irr}(G \mid \theta)$. Identify $G^{*}$ with $G$ and let $s \in G$ be a semisimple element such that $\chi$ belongs to the rational 
Lusztig series $\mathcal{E}(G,(s))$ labeled by $s^{G}$. Then one of the following statements holds for $C:=\mathbf{C}_{G}(s)$.

(a) $\chi(1)=\theta(1)$ and $C \cong \prod_{i=1}^{m} G U_{k_{i}}(q)$ with $m>1, k_{1}<\ldots<k_{m}$, and $\sum_{i=1}^{m} k_{i}=n$. Moreover, $s$ is $G$-conjugate to

$$
\operatorname{diag}(\underbrace{\alpha_{1}, \ldots, \alpha_{1}}_{k_{1}}, \ldots, \underbrace{\alpha_{m}, \ldots, \alpha_{m}}_{k_{m}})
$$

for some pairwise distinct $\alpha_{1}, \ldots, \alpha_{m} \in \mu_{q+1}$.

(b) $\chi(1)=2 \theta(1), C \cong G U_{k}(q)^{2}, n=2 k$, and $2 \mid k$. Moreover, $s$ is $G$-conjugate to

$$
\operatorname{diag}(\underbrace{\alpha, \ldots, \alpha}_{k}, \underbrace{-\alpha, \ldots,-\alpha}_{k})
$$

for some $\alpha \in \mu_{q+1}$.

Proof. As in the proof of Lemma 4.5, we have that $2(q+1) \nmid \chi(1)$. Next,

$$
C:=\mathbf{C}_{G}(s) \cong \prod_{i=1}^{u} G U_{k_{i}}\left(q^{a_{i}}\right) \times \prod_{j=1}^{v} G L_{l_{j}}\left(q^{2 b_{j}}\right),
$$

where $k_{i}, l_{j}, a_{i}, b_{j} \geq 1$ are integers, $2 \nmid a_{i}$, and $\sum_{i=1}^{u} k_{i} a_{i}+2 \sum_{j=1}^{v} l_{j} b_{j}=n$. Furthermore, $\chi(1) / \theta(1)=|J|$, where $J:=\{\lambda \in \operatorname{Irr}(G / L) \mid \lambda \chi=\chi\}$. Again, there is a group isomorphism $z \mapsto \lambda_{z}$ between $\mathbf{Z}(G)$ and $\operatorname{Irr}(G / L)$ such that the multiplication by $\lambda_{z}$ sends the rational Lusztig series $\mathcal{E}(G,(s))$ to $\mathcal{E}(G,(s z))$, and $s$ and $s z$ are conjugate in $G$ whenever $\lambda_{z} \in J$. Arguing as in (i) of the proof of Lemma 4.5, we see that $a_{i}=1$ for all $i$.

Suppose that $v>0$. Note that

$$
\left[G U_{2 l b}(q): G L_{l}\left(q^{2 b}\right)\right]_{p^{\prime}}=\prod_{1 \leq i<2 l b, 2 b \nmid i}\left(q^{i}-(-1)^{i}\right)
$$

is divisible by $(q+1)^{2}$ if $l b \geq 2$. Hence the condition $2(q+1) \nmid \chi(1)$ implies that $l_{j} b_{j}=1$ for all $j$. If moreover $v \geq 2$, then

$$
\left[G U_{2 l_{1} b_{1}+2 l_{2} b_{2}}(q): G L_{l_{1}}\left(q^{2 b_{1}}\right) \times G L_{l_{2}}\left(q^{2 b_{2}}\right)\right]_{p^{\prime}}=\left[G U_{4}(q): G L_{1}\left(q^{2}\right) \times G L_{1}\left(q^{2}\right)\right]_{p^{\prime}}
$$

is again divisible $(q+1)^{2}$, a contradiction. Thus $v=1$, and, as $n>2$, we see that $s$ is $G$-conjugate to

$$
\operatorname{diag}(\underbrace{\alpha_{1}, \ldots, \alpha_{1}}_{k_{1}}, \ldots, \underbrace{\alpha_{m}, \ldots, \alpha_{m}}_{k_{m}}, \beta, \beta^{-q})
$$

where $\beta \in \mathbb{F}_{q^{2}}^{\times} \backslash \mu_{q+1}$, and $\alpha_{1}, \ldots, \alpha_{m} \in \mu_{q+1}$ are pairwise distinct. Since

$$
\left[G U_{2 l_{1} b_{1}}(q): G L_{l_{1}}\left(q^{2 b_{1}}\right)\right]_{p^{\prime}}=\left[G U_{2}(q): G L_{1}\left(q^{2}\right)\right]_{p^{\prime}}=q+1
$$

is even and $2 \nmid \theta(1)=\chi(1) /|J|$, we must have that $|J|$ is even. It follows that $J \ni \lambda_{-1_{V}}$ (where $V=\mathbb{F}_{q^{2}}^{n}$ is the natural $G$-module), and so $s$ and $-s$ are $G$-conjugate. Thus, 
the multiplication by -1 acts on the multi-set $\operatorname{Spec}(s)$, and this action leaves $\mu_{q+1}$ invariant. Renaming the $\alpha_{i}$ 's if necessary, we may therefore assume that $\alpha_{2}=-\alpha_{1}$ and $k_{2}=k_{1}$. By Lemma 4.3(i) we have that

$$
\left[G U_{k_{1} a_{1}+k_{2} a_{2}}(q): G U_{k_{1}}\left(q^{a_{1}}\right) \times G U_{k_{2}}\left(q^{a_{2}}\right)\right]_{p^{\prime}}
$$

is even. Together with (4.2), this implies that $2(q+1) \nmid[G: C]_{p^{\prime}}$, a contradiction.

We have shown that $v=0$ and $a_{i}=1$ for all $i$. Now we can argue as in part (iii) of the proof of Lemma 4.5.

Lemma 4.7. Let $S=P S L_{n}^{\epsilon}(q)$ and $H=P G L_{n}^{\epsilon}(q)$ with $n \geq 3, \epsilon= \pm$, and $q=p^{f}$ for some odd prime $p$. Let $\theta \in \operatorname{Irr}_{2^{\prime}}(S)$ be non-unipotent and let $\chi \in \operatorname{Irr}(H \mid \theta)$. If $s \in H^{*} \cong L=S L_{n}^{\epsilon}(q)$ is a semisimple element such that $\chi$ belongs to the rational Lusztig series $\mathcal{E}(H,(s))$ labeled by $s^{H^{*}}$, then one of the following statements holds.

(a) $\chi(1)=\theta(1)$ and $s$ is $H^{*}$-conjugate to

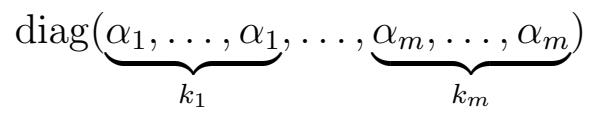

for some pairwise distinct $\alpha_{1}, \ldots, \alpha_{m} \in \mu_{q-\epsilon}$, where $m>1, k_{1}<\ldots<k_{m}$, and $\sum_{i=1}^{m} k_{i}=n$.

(b) $\chi(1)=2 \theta(1)$ and $s$ is $H^{*}$-conjugate to

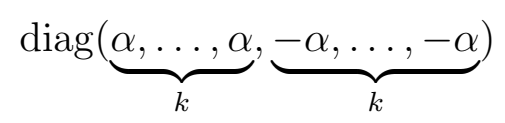

for some $\alpha \in \mu_{q-\epsilon}$, where $2 \mid k=n / 2$.

Proof. We can identify $H^{*}$ with $L$ and set $G=G L_{n}^{\epsilon}(q)$. Again, as $H / S$ is cyclic of order dividing $q-\epsilon$ and $2 \nmid \theta(1), 2(q-\epsilon) \nmid \chi(1)$. Also, $\chi(1) / \theta(1)=|J|$, where $J:=\{\lambda \in \operatorname{Irr}(H / S) \mid \lambda \chi=\chi\}$, and $J$ can be embedded in $\mathbf{Z}(L)$ via $\lambda_{z} \mapsto z$. As in the proof of Lemma $4.5, s$ and $s z$ are $L$-conjugate whenever $\lambda_{z} \in J$. Note that $s$ is contained in a maximal torus $T$ of $G$, and $L T=G$, whence $L \mathbf{C}_{G}(s)=G$. It follows that $\left[L: \mathbf{C}_{L}(s)\right]_{p^{\prime}}=\left[G: \mathbf{C}_{G}(s)\right]_{p^{\prime}}$. Now we can view $\chi$ as a character of $G$ and repeat the proofs of Lemmas 4.5 and 4.6 verbatim to identify possible $s$.

Recall [GLS, Theorem 2.5.12] that, if $S=P S L_{n}^{\epsilon}(q)$ (with $n \geq 3, q=p^{f}, p$ a prime, and $(n, q, \epsilon) \neq(3,2,-))$, then $\operatorname{Aut}(S) \cong H / S \rtimes D$, where $H=P G L_{n}^{\epsilon}(q)$, and the abelian group $D$ of order $2 f$ is generated by graph and field automorphisms (as specialized eg. in $[\mathrm{CS} 2, \S 3.2])$. In particular, $D$ contains an automorphism $\varphi=\nu \tau$, where $\tau(g)={ }^{t} g^{-1}$ and $\nu$ is an involutory inner automorphism of $S$.

Lemma 4.8. Suppose we are in case (i) of Lemma 4.1 and moreover conclusion (b) of Lemma 4.7 holds. Then $\theta$ extends to $R$.

Proof. Recall that $\chi \in \operatorname{Irr}(H \mid \theta)$ is labeled by the semisimple conjugacy class $s^{L}$ with $s$ described in Lemma $4.7(\mathrm{~b})$ and a unipotent character $\psi$ of $\mathbf{C}_{L}(s)=\left(G L_{k}^{\epsilon}(q) \times\right.$ 
$\left.G L_{k}^{\epsilon}(q)\right) \cap L$ (where we have again identified $H^{*}$ with $L$ ). Next, $D$ also acts on $H$ and $L$. As $H$ is of adjoint type, the Jordan decomposition for $\operatorname{Irr}(H)$ can be chosen to be equivariant under $D$, see [CS1, Theorem 3.1]. We may assume that $R \leq \tilde{R}$, where $\tilde{R} / S \in \operatorname{Syl}_{r}(\operatorname{Out}(S))$ and $\tilde{R}$ contains the unique Sylow $r$-subgroup $D_{1}$ of $D$. Note that $D_{1}$ is cyclic, consists of field automorphisms, and $D_{1}$ induces field automorphisms of $\mathbf{C}_{L}(s)$.

Now $1=\operatorname{det}(s)=\alpha^{n}$, and so $\alpha^{d}=1$ for $d:=\operatorname{gcd}(n, q-\epsilon)=|\mathbf{Z}(L)|$. In particular, $z:=\alpha^{-1} \cdot 1_{V} \in \mathbf{Z}(L)$, where $V$ is again the natural $L$-module. Multiplying $\chi$ by $\lambda_{z} \in \operatorname{Irr}(H / S)$, we may assume that $\alpha=1$. But now, $s^{L}$ is fixed by $D_{1}$. On the other hand, $\psi$ is also fixed by field automorphisms, cf. for instance [M2, Theorem 2.5]. The $D$-equivariance of the Jordan decomposition now implies that $\chi$ is $D_{1}$-invariant, and so $\chi$ extends to a character $\tilde{\chi}$ of $H D_{1} \geq \tilde{R}$. We have shown that $\tilde{\chi}_{R}$ is a character of degree $2 \theta(1)$ that lies above $\theta$. On the other hand, as $2 \nmid|R / S|$, any irreducible character of $R$ lying above $\theta$ has degree an odd multiple of $\theta(1)$. It follows that $\tilde{\chi}_{R}$ is the sum of two irreducible characters of $R$, both of degree $\theta(1)$, and (at least) one of them extends $\theta$.

Lemma 4.9. Suppose we are in case (i) of Lemma 4.1 and moreover conclusion (a) of Lemma 4.7 holds. Assume in addition that $\theta$ is $\tau$-invariant, where $\tau$ is the transpose-inverse automorphism. Then $\theta$ extends to $R$.

Proof. Fix some $\chi \in \operatorname{Irr}(H \mid \theta)$ and let $G=G L_{n}^{\epsilon}(q), Z=\mathbf{Z}(G)$, so that $H=G / Z$. It is convenient to view $\theta$ as an $Z L$-character (as $Z L / Z \cong S$ ) and view $\chi$ as a $G$ character. As $\chi_{L}=\theta$, case (a) of Lemma 4.5, respectively of Lemma 4.6, occurs. Note that $\tau$ acts on $L$ and $G$ as well. Hence, $\tau$ acts on

$$
\operatorname{Irr}(G \mid \theta)=\{\chi \lambda \mid \lambda \in \operatorname{Irr}(G / L)\} .
$$

By [CS1, Theorem 3.2], we may assume that the Jordan decomposition in consideration is $D$-equivariant. As in the proof of Lemma 4.5, we can write $\operatorname{Irr}(G / L)=\left\{\lambda_{z} \mid\right.$ $z \in Z\}$, and, if $\chi \in \mathcal{E}(G,(s))$ then $\chi \lambda_{z} \in \mathcal{E}(G,(s z))$. Now we can write $\chi^{\tau}=\chi \lambda_{z}$ for some $z=\gamma \cdot 1_{V}$, where $\gamma \in \mu_{q-\epsilon}$ and $V$ is the natural $G$-module. The $D$-equivariance of the Jordan decomposition implies that $\chi^{\tau} \in \mathcal{E}\left(G,\left(s^{\tau}\right)\right.$ ) (note that $\tau=\nu \varphi$ with $\nu \in \operatorname{Inn}(G)$ and $\varphi \in D)$. It follows that $s^{\tau}$ and $\gamma s$ are $G$-conjugate and so the multiplication by $\gamma$ sends the multi-set $\operatorname{Spec}(s)$, containing $\alpha_{i}$ with multiplicity $k_{i}$, where $\alpha_{i} \in \mu_{q-\epsilon}, 1 \leq i \leq m$, and $k_{1}<\ldots<k_{m}$, to $\operatorname{Spec}\left(s^{\tau}\right)=\operatorname{Spec}\left(s^{-1}\right)$. Hence, $\gamma \alpha_{i}=\alpha_{i}^{-1}$ and

$$
\alpha_{i}^{2}=\gamma^{-1}
$$

for all $i$. Recall that $\theta$ is non-unipotent as an $S$-character, so $m>1$. Furthermore, $\alpha_{1}, \ldots, \alpha_{m}$ are pairwise distinct. Hence (4.3) implies that $m=2$, and $\alpha_{2}=-\alpha_{1}$.

As $2 \nmid \theta(1)=\chi(1)=[G: C]_{p^{\prime}} \psi(1)$, where $\psi$ is a unipotent character of $C:=\mathbf{C}_{G}(s)$, Lemma 4.3(ii) implies that at least one of $k_{1}$ and $k_{2}$ must be even. Renaming the 
$\alpha_{i}$ 's if necessary, we may assume that $2 \mid k_{2}$. Now we consider

$$
\chi^{*}=\chi \lambda_{\alpha_{1}^{-1} \cdot 1_{V}} \in \operatorname{Irr}(G \mid \theta)
$$

that belongs to the Lusztig series $\mathcal{E}\left(G,\left(s^{*}\right)\right)$ and corresponds to the unipotent character $\psi^{*}$ of $\mathbf{C}_{G}\left(s^{*}\right)=C$, where

$$
s^{*}:=s \alpha_{1}^{-1}=\operatorname{diag}(\underbrace{1,1, \ldots, 1}_{k_{1}}, \underbrace{-1,-1, \ldots,-1}_{k_{2}}) .
$$

Note that $s^{*} \in L=[G, G]$. This in turn implies by [NT2, Lemma 4.4(ii)] that $\chi^{*}$ is trivial at $Z$ and so can be viewed as an $H$-character. Again by [M2, Theorem 2.5], the unipotent character $\psi^{*}$ of $C \cong G L_{k_{1}}^{\epsilon}(q) \times G L_{k_{2}}^{\epsilon}(q)$ is invariant under field automorphisms. Now we can finish as in the proof of Lemma 4.8. (Alternatively, we may also observe that $\chi^{*}$ is invariant under $\varphi$ and $\tau$. As $g$ and ${ }^{t} g$ are conjugate in $\mathcal{G}:=G L_{n}\left(\overline{\mathbb{F}}_{q}\right)$ and $\mathbf{C}_{\mathcal{G}}(g)$ is connected, it follows by the Lang-Steinberg theorem that $g^{-1}$ and $\tau(g)={ }^{t} g^{-1}$ are conjugate in $G$. Hence the $\tau$-invariance of $\chi^{*}$ now implies that $\chi^{*}=\overline{\chi^{*}}$ and so $\theta$ is real.)

Lemma 4.10. Let $q=p^{f}$ for some odd prime $p$ and some non-2-power $f$. Let the abelian group $D=\langle\varsigma, j\rangle$ act on $F:=\mathbb{F}_{q}^{\times}$via $\varsigma(x)=x^{p}$ and $j(x)=x^{-1}$. If $E \leq D$ is a 2-subgroup not containing $j$, then $\left|\mathbf{C}_{\mathbf{O}_{2^{\prime}}(F)}(E)\right| \geq 7$.

Proof. Write $f=2^{a} f_{0}$ where $f_{0}$ is odd, and let $q_{0}=p^{f_{0}}$. Note that $f_{0} \geq 3$ as $f \neq 2^{a}$, whence $\left(q_{0}-1\right) /(p-1) \geq 13$ and $\left(q_{0}+1\right) /(p+1) \geq 7$. Suppose that $E \leq\langle\varsigma\rangle$. Then $E \leq\left\langle\varsigma^{f_{0}}\right\rangle$ acts trivially on the subgroup $\left\{x \in F \mid x^{\left(q_{0}-1\right) /(p-1)}=1\right\}$ of $\mathbf{O}_{2^{\prime}}(F)$.

Suppose now that $E \not \leq\langle\varsigma\rangle$. Since $E \not \ngtr j$ by assumption, we must have that

$$
E=\left\langle\varsigma^{2^{b}} f_{0} j\right\rangle
$$

for some $0 \leq b \leq a-1$. If $b=0$, then $E$ acts trivially on the subgroup $\{x \in$ $\left.F \mid x^{\left(q_{0}+1\right) /(p+1)}=1\right\}$ of $\mathbf{O}_{2^{\prime}}(F)$. If $b \geq 1$, then $E$ acts trivially on the subgroup $\left\{x \in F \mid x^{\left(q_{0}^{2^{b}}+1\right) / 2}=1\right\}$ of $\mathbf{O}_{2^{\prime}}(F)$.

Proposition 4.11. Suppose we are in case (i) of Lemma 4.1. Then $\theta$ extends to $R$.

Proof. (i) As usual, we identify $H^{*}$ with $L=S L_{n}^{\epsilon}(q)$. At the same time we will view $S$ as $L Z / Z$ with $G:=G L_{n}^{\epsilon}(q)$ and $Z:=\mathbf{Z}(G)$. Let $V=\mathbb{F}_{q}^{n}$, respectively $\mathbb{F}_{q^{2}}^{n}$, denote the natural $G$-module for $\epsilon=+$, respectively for $\epsilon=-$. We can also identify $A=\operatorname{Aut}(S)$ with $(G \rtimes D) / Z$. By Proposition 4.2 and Lemma 4.8, we may assume that $p$ is odd and that conclusion (a) of Lemma 4.7 holds.

Let $D_{2}$ denote the unique Sylow 2-subgroup of $D$. We will choose $\tilde{P} \in \operatorname{Syl}_{2}(G \rtimes D)$ containing $D_{2}$ as follows. Let

$$
n=2^{a_{1}}+2^{a_{2}}+\ldots+2^{a_{t}}
$$


be the 2-adic decomposition of $n$, with $a_{1}>a_{2}>\ldots>a_{t} \geq 0$. Note that $t \geq 2$, as otherwise $\left[L: \mathbf{C}_{L}(s)\right]_{p^{\prime}}$ is even by Lemma 4.4 , contrary to the assumption that $2 \nmid \theta(1)=\chi(1)$. Now we can decompose

$$
V=V_{1} \oplus V_{2} \oplus \ldots \oplus V_{t}
$$

into a direct (orthogonal if $\epsilon=-$ ) sum of subspaces, where $\operatorname{dim} V_{i}=2^{a_{i}}$. We can define $\tau: g \mapsto{ }^{t} g^{-1}$ by writing $g \in G$ in a basis consistent with this decomposition. Furthermore, we can choose the involutory automorphism $\nu$ to be induced by an element of $L$ fixing this decomposition. We also choose $\sigma \in D$ to be the automorphism by raising each entry of the matrix of $g \in G$ in this basis to its $p$-th power. Then $\varphi=\nu \tau$ and $\sigma$ act on each $G L^{\epsilon}\left(V_{i}\right)$. Now, we can choose a $D_{2}$-invariant Sylow 2-subgroup $Q_{i}$ of $G L^{\epsilon}\left(V_{i}\right)$. Note that $\tilde{Q}:=Q_{1} \times \ldots \times Q_{t} \in \operatorname{Syl}_{2}(G)$; in particular,

$$
Q=\left(Q_{1} \times Q_{2} \times \ldots \times Q_{t}\right) \cap L \in \operatorname{Syl}_{2}(L) .
$$

Now we can extend $\tilde{Q}$ (first to a Sylow 2-subgroup of $\mathbf{N}_{G \rtimes D_{2}}(\tilde{Q})$, and then) to a Sylow 2-subgroup $\tilde{P}$ of $G \rtimes D$ that contains $D_{2}$. In fact, $\tilde{P}=\tilde{Q} \rtimes D_{2}$.

(ii) Recall that, by the assumption in Theorem 3.1, $X / S \in \operatorname{Syl}_{2}(I / S)$ for $I=I_{A}(\theta)$ and $X$ has a self-normalizing Sylow 2-subgroup $\bar{P}$. Since conclusion (a) of Lemma 4.7 holds, we have that $I \geq H / S \triangleleft A / S$. Replacing $\theta$ by a suitable $A$-conjugate, we may assume that $\bar{P}=P Z / Z$, where the Sylow 2-subgroup $P$ of the full inverse image of $I$ in $G \rtimes D$ is chosen such that $P \leq \tilde{P}$. As $P \geq \tilde{Q}$ and $\tilde{P}=\tilde{Q} \rtimes D_{2}$, we can write $P=\tilde{Q} \rtimes E$ for some subgroup $E \leq D_{2}$. Furthermore, by Lemma 4.9 we may assume that $I \not \supset \tau$, and so $E \not \supset \varphi$.

As $\bar{P}$ is self-normalizing in $X$,

$$
\mathbf{C}_{\mathbf{N}_{S}(\bar{Q}) / \bar{Q}}(\bar{P})=1
$$

by [NTT, Lemma 2.1(ii)], where $\bar{Q}:=Q Z / Z$. We now show that our assumptions imply $t=2$. Indeed, suppose that $t \geq 3$. The construction of $Q$ ensures that $P \cap G=\tilde{Q}$ is centralized by

$$
Y:=\left\{\operatorname{diag}\left(\beta_{1} \cdot 1_{V_{1}}, \ldots \beta_{t} \cdot 1_{V_{t}}\right) \mid \beta_{i} \in \mu_{q-\epsilon}\right\} \cap L .
$$

Assume that $\epsilon=-$. Then we can choose $\beta \in \mu_{q+1}$ of odd order $(q+1)_{2^{\prime}}>1$, so that

$$
h(\beta):=\operatorname{diag}\left(\beta \cdot 1_{V_{1}}, 1_{V_{2}}, \ldots, 1_{V_{t-1}}, \beta^{-2^{a_{1}-a_{t}}} \cdot 1_{V_{t}}\right)
$$

has odd order and belongs to $\mathbf{C}_{L}(\tilde{Q}) \backslash Z$. Now the condition (4.4) implies that $P>\tilde{Q}$, and so $I>H$. But $\operatorname{Aut}(S) / H \cong D \cong C_{2 f}$ (as $\left.\epsilon=-\right)$ and $\varphi$ is the unique involution in $D$. Hence we conclude that $I \ni \varphi$ and so $I \ni \tau$, contrary to our assumption. In fact, by considering the element $h(\beta)$ for $t=2$ and using the condition $I \not \supset \tau$, we see that $h(\beta) \in Z \cap L=\mathbf{Z}(L)$, and so

$$
(q+1)_{2^{\prime}} \mid n .
$$

We will need this observation later. 
Consider the case $\epsilon=+$. Then $D=\langle\sigma, \varphi\rangle$. By our construction,

$$
\sigma: \beta \cdot 1_{V_{i}} \mapsto \beta^{p} \cdot 1_{V_{i}}, \varphi: \beta \cdot 1_{V_{i}} \mapsto \beta^{-1} \cdot 1_{V_{i}}
$$

for $\beta \in \mathbb{F}_{q}^{\times}$. Thus $\sigma$ and $\varphi$ induce on $\mathbb{F}_{q}^{\times}$the maps $\varsigma$ and $j$ of Lemma 4.10. By our assumption, $P=\tilde{Q} \rtimes E$ with $E \not \supset \varphi$. Hence, by Lemma 4.10, we can choose $1 \neq \beta \in \mathbf{C}_{\mathbf{O}_{2^{\prime}}\left(\mathbb{F}_{q}^{\times}\right)}(E)$ such that

$$
\operatorname{diag}\left(\beta \cdot 1_{V_{1}}, 1_{V_{2}}, \ldots, 1_{V_{t-1}}, \beta^{-2^{a_{1}-a_{t}}} \cdot 1_{V_{t}}\right)
$$

has odd order and belongs to $\mathbf{C}_{L}(P) \backslash Z$, contradicting the condition (4.4).

(iii) We have shown that $t=2$, i.e. $n=2^{u+v}+2^{v}$, with $u \geq 1$ and $v \geq 0$. Now, we view $\theta$ as a character of $L Z$ that is trivial at $Z$ and consider $\chi \in \operatorname{Irr}(G \mid \theta)$ belonging to $\mathcal{E}\left(G,(s)\right.$ ) (and identify $G^{*}$ with $G$ ). Since the condition 4.7 (a) holds, we are in case (a) of Lemma 4.5 when $\epsilon=+$ and of Lemma 4.6 when $\epsilon=-$. As $2 \nmid \chi(1)=\theta(1)$, $\left[G: \mathbf{C}_{G}(s)\right]$ is odd. In particular, $2 \nmid\left[G L_{n}^{\epsilon}(q): G L_{n-k_{i}}^{\epsilon}(q) \times G L_{k_{i}}(q)\right]$ for all $i$. Since $n=2^{u+v}+2^{v}$, this implies by Lemma 4.4 that $k_{i} \in\left\{2^{u+v}, 2^{v}\right\}$. Hence $m=2$ and $s$ is $G$-conjugate to

$$
\operatorname{diag}(\underbrace{\alpha, \alpha, \ldots, \alpha}_{2^{u+v}}, \underbrace{\alpha \gamma, \alpha \gamma, \ldots, \alpha \gamma}_{2^{v}})
$$

where $\alpha, \gamma \in \mu_{q-\epsilon}$. Furthermore, $Z \leq \operatorname{Ker}(\chi)$ implies by [NT2, Lemma 4.4(ii)] that

$$
1=\operatorname{det}(s)=\alpha^{n} \gamma^{2^{v}} \text {. }
$$

Recall that $r \mid f$, so we will write $f=2^{a} r^{c} f_{0}$ with $\operatorname{gcd}\left(f_{0}, 2 r\right)=1$. In what follows we may assume by [CS1, Theorem 3.1] that the Jordan decomposition for $\operatorname{Irr}(G)$ is $D$-equivariant. Changing the notation, we will view $I$ as $I_{G \rtimes D}(\theta)$. Let $\tilde{R}$ be the complete inverse image of $R$ in $G \rtimes D$.

(iv) Now we can complete the case $\epsilon=-$. In this case, $D=\langle\sigma\rangle \cong C_{2 f}$. Since $I \geq G$ and $\tilde{R} / L Z$ is non-cyclic, we have that $\tilde{R} G=G\left\langle\sigma_{1}\right\rangle$, where $\sigma_{1}=\sigma^{2^{a+1} r^{d} f_{0}}$ for some $d<c$. Set $q_{1}=p^{2^{a} r^{d} f_{0}}$ so that $q=q_{1}^{r^{c-d}}$ and $\sigma_{1}(x)=x^{q_{1}^{2}}$ for $x \in \overline{\mathbb{F}}_{p}$. As $\sigma_{1}$ fixes $\theta$, it acts on

$$
\operatorname{Irr}(G \mid \theta)=\left\{\chi \lambda_{z} \mid z \in Z\right\}
$$

and so sends $\chi$ to say $\chi \lambda_{z}$ for some $z=\delta \cdot 1_{V}$. Now $\chi \in \mathcal{E}(G,(s)), \chi \lambda_{z} \in \mathcal{E}(G,(\delta s))$, and $\sigma_{1}$ sends $\mathcal{E}(G,(s))$ to $\mathcal{E}\left(G,\left(\sigma_{1}(s)\right)\right)$. It follows that $\sigma_{1}(s)$ and $\delta s$ are $G$-conjugate. Inspecting the multi-sets of eigenvalues, we then see that

$$
\alpha^{q_{1}^{2}}=\delta \alpha,(\alpha \gamma)^{q_{1}^{2}}=\delta \alpha \gamma
$$

and so $\gamma^{q_{1}^{2}-1}=1$. As $\gamma \in \mu_{q+1}$ and $q=q_{1}^{r^{c-d}}$, in fact we have

$$
\gamma^{q_{1}+1}=1 \text {. }
$$

On the other hand, $n=n^{\prime}(q+1)_{2^{\prime}}$ for some $n^{\prime} \in \mathbb{Z}$ by (4.5). Hence (4.6) implies that

$$
\gamma^{2^{v}(q+1)_{2}}=\alpha^{-n(q+1)_{2}}=\alpha^{-n^{\prime}(q+1)}=1 \text {, }
$$


and so $|\gamma|$ is a 2-power. Together with (4.7), we have shown that $\gamma^{\left(q_{1}+1\right)_{2}}=1$. Furthermore, $\operatorname{gcd}\left(n, q_{1}+1\right)_{2} \mid n_{2}=2^{v}$, so

$$
\left(\gamma^{2^{v}}\right)^{\frac{\left(q_{1}+1\right)_{2}}{\operatorname{gcd}\left(n, q_{1}+1\right)_{2}}}=1 \text {. }
$$

In particular, $\left(\gamma^{2^{v}}\right)^{\left(q_{1}+1\right) / \operatorname{gcd}\left(n, q_{1}+1\right)}=1$, and so we can find $\varepsilon \in \mu_{q_{1}+1}<\mu_{q+1}$ such that

$$
\gamma^{-2^{v}}=\varepsilon^{n}
$$

Setting $\chi^{*}=\chi \lambda_{t}$ with $t:=(\varepsilon / \alpha) \cdot 1_{V}$, we have that $\chi^{*} \in \mathcal{E}\left(G,\left(s^{*}\right)\right)$, where

$$
s^{*}=\operatorname{diag}(\underbrace{\varepsilon, \varepsilon, \ldots, \varepsilon}_{2^{u+v}}, \underbrace{\varepsilon \gamma, \varepsilon \gamma, \ldots, \varepsilon \gamma}_{2^{v}}) \text {. }
$$

The choice (4.8) yields that $\operatorname{det}\left(s^{*}\right)=1$, and so $Z \leq \operatorname{Ker}\left(\chi^{*}\right)$ by [NT2, Lemma 4.4(ii)]. Moreover, both $\varepsilon$ and $\gamma$ belong to $\mu_{q_{1}+1}$, see (4.7), and so are fixed by $\sigma_{1}$. Since unipotent characters of $\mathbf{C}_{G}\left(s^{*}\right)$ are $\sigma_{1}$-invariant, we conclude that $\chi^{*}$ is $\sigma_{1}$-invariant and so extends to a character of $G\left\langle\sigma_{1}\right\rangle=\tilde{R} G$ that is trivial at $Z$. Consequently, $\theta$ extends to $R$, as desired.

(v) Now we handle the case $\epsilon=+$. Since $I \geq G$ and $\tilde{R} / L Z$ is non-cyclic, we now have that $\tilde{R} G=G\left\langle\sigma_{1}\right\rangle$, where $\sigma_{1}=\sigma^{2^{a} r^{d}} f_{0}$ for some $d<c$. We again set $q_{1}=p^{2^{a} r^{d}} f_{0}$ so that $\sigma_{1}(x)=x^{q_{1}}$ for $x \in \overline{\mathbb{F}}_{p}$. As $\sigma_{1}$ fixes $\theta$, it acts on

$$
\operatorname{Irr}(G \mid \theta)=\left\{\chi \lambda_{z} \mid z \in Z\right\}
$$

and so sends $\chi$ to say $\chi \lambda_{z}$ for some $z=\delta \cdot 1_{V}$. As in (iv), we then see that $\sigma_{1}(s)$ and $\delta s$ are $G$-conjugate and so

$$
\alpha^{q_{1}}=\delta \alpha,(\alpha \gamma)^{q_{1}}=\delta \alpha \gamma
$$

It follows that

$$
\gamma^{q_{1}-1}=1
$$

Recall that $P=\tilde{Q} \rtimes E$ with $\varphi \notin E \leq D_{2}$. Hence we can write $E=\left\langle\sigma_{2} \varphi^{j}\right\rangle$, where $\sigma_{2}=\sigma^{2^{b} r^{c} f_{0}}$ with $0 \leq b \leq a$ and $j \in\{0,1\}$. Set $q_{2}:=p^{2^{b} r^{c} f_{0}}$ and $\kappa=(-1)^{j}$. Note that $\sigma_{2} \varphi^{j}$ sends $\beta \cdot 1_{V_{i}}$ to $\beta^{\kappa q_{2}} \cdot 1_{V_{i}}$ for any $\beta \in \mu_{q-1}$. Moreover, in the case $j=1$, we must have that $b<a$ as $E \not \supset \varphi$, and so $\left(q_{2}+1\right) \mid(q-1)$. We certainly have $\left(q_{2}-1\right) \mid(q-1)$ if $j=0$. Thus $\left(q_{2}-\kappa\right) \mid(q-1)$ in either case. Now, for any $\beta \in \mu_{q_{2}-\kappa} \leq \mu_{q-1}$ of odd order, the element $\operatorname{diag}\left(\beta \cdot 1_{V_{1}}, \beta^{-2^{u}} \cdot 1_{V_{2}}\right)$ of $L$ centralizes $P$ and so must belong to $Z$ by (4.4). It follows that $\beta=\beta^{-2^{u}}$ for any such $\beta$, whence $\left(q_{2}-\kappa\right)_{2^{\prime}} \mid n$ and we can write

$$
n=2^{v} n_{\kappa}\left(q_{2}-\kappa\right)_{2^{\prime}}
$$


for some odd $n_{\kappa} \in \mathbb{N}$. Arguing as above, we also see that the $\sigma_{2} \varphi^{j}$-invariance of $\theta$ implies that

$$
\gamma^{q_{2}-\kappa}=1
$$

Using (4.6) and (4.11), we now obtain that

$$
\left(\gamma^{2^{v}}\right)^{\frac{q-1}{q_{2}-\kappa} \cdot \max \left(1,2^{-v}\left(q_{2}-\kappa\right)_{2}\right)}=\alpha^{-n_{\kappa} \cdot \frac{q-1}{q_{2}-\kappa} \cdot \max \left(2^{v},\left(q_{2}-\kappa\right)_{2}\right) \cdot\left(q_{2}-\kappa\right)_{2^{\prime}}}=1 .
$$

Hence, $\left|\gamma^{2^{v}}\right|_{2^{\prime}}$ divides

$$
\frac{q-1}{q_{2}-\kappa}=\frac{q_{2}^{2^{a-b}}-1}{q_{2}-\kappa} \equiv 2^{a-b} \kappa\left(\bmod \left(q_{2}-\kappa\right)\right) .
$$

This implies by (4.12) that $|\gamma|$ is a 2-power, and (4.10) now yields that $\gamma^{\left(q_{1}-1\right)_{2}}=1$. But $\operatorname{gcd}\left(n, q_{1}-1\right)_{2} \mid n_{2}=2^{v}$, so $\left(\gamma^{2^{v}}\right)^{\left(q_{1}-1\right)_{2} / \operatorname{gcd}\left(n, q_{1}-1\right)_{2}}=1$. It follows that

$$
\left(\gamma^{2^{v}}\right)^{\frac{q_{1}-1}{\operatorname{gcd}\left(n, q_{1}-1\right)}}=1
$$

and so we can find $\varepsilon \in \mu_{q_{1}-1}<\mu_{q-1}$ such that (4.8) holds. Setting $\chi^{*}=\chi \lambda_{t}$ with $t:=(\varepsilon / \alpha) \cdot 1_{V}$, we have that $\chi^{*} \in \mathcal{E}\left(G,\left(s^{*}\right)\right)$, where $s^{*}$ is defined in (4.9). The choice (4.8) again yields that $\operatorname{det}\left(s^{*}\right)=1$, and so $Z \leq \operatorname{Ker}\left(\chi^{*}\right)$. Moreover, both $\varepsilon$ and $\gamma$ belong to $\mu_{q_{1}-1}$, see $(4.10)$, and so are fixed by $\sigma_{1}$. Since unipotent characters of $\mathbf{C}_{G}\left(s^{*}\right)$ are $\sigma_{1}$-invariant, we conclude that $\chi^{*}$ is $\sigma_{1}$-invariant and so extends to a character of $G\left\langle\sigma_{1}\right\rangle=\tilde{R} G$ that is trivial at $Z$. Consequently, $\theta$ extends to $R$, as desired.

4.3. Groups of type $E_{6}$. To handle case (ii) of Lemma 4.1, we will need the following variant of Burnside's fusion lemma:

Lemma 4.12. Let $G$ be a finite group with a normal subgroup $K, p$ a prime, and let $Q \in \operatorname{Syl}_{p}(K)$. Then $\mathbf{N}_{G}(Q)$ controls $G$-fusion of elements in $\mathbf{Z}\left(\mathbf{N}_{K}(Q)\right)$. Moreover, if $G=K E$ for some $E \leq G$ and $Q$ is E-invariant, then whenever $y=x^{g}$ for some $x, y \in \mathbf{Z}\left(\mathbf{N}_{K}(Q)\right)$ and $g \in G$, we can find $d \in E$ such that $y=x^{d}$ and $\langle K, g\rangle=\langle K, d\rangle$.

Proof. Suppose that $y=x^{g}$ for some $x, y \in \mathbf{Z}\left(\mathbf{N}_{K}(Q)\right)$ and $g \in G$. Then $Q^{g}$ and $Q$ are Sylow $p$-subgroups of $\mathbf{C}_{K}(y)$, and so $Q=Q^{g c}$ for some $c \in \mathbf{C}_{K}(y)$. Now $g c \in \mathbf{N}_{G}(Q)$ and $y=y^{c}=x^{g c}$, whence the first statement follows.

For the second statement, we have $\mathbf{N}_{G}(Q)=\mathbf{N}_{K}(Q) E$ by assumption. So we can write $g c=n d$ for some $n \in \mathbf{N}_{K}(Q)$ and $d \in E$. Now we have $x=x^{n}$ as $x \in \mathbf{Z}\left(\mathbf{N}_{K}(Q)\right)$, and so

$$
y=y^{c}=x^{g c}=x^{n d}=x^{d} .
$$

Furthermore,

$$
\langle K, d\rangle=\left\langle K, n^{-1} g c\right\rangle \leq\langle K, g\rangle=\left\langle K, n d c^{-1}\right\rangle \leq\langle K, d\rangle
$$

and so we are done. 
Lemma 4.13. Let $p$ be an odd prime, $q=p^{f} \equiv \epsilon(\bmod 3)$ for some $\epsilon= \pm$.

(i) Let $S$ be the simple group of type $E_{6}^{\epsilon}(q)$ and $Q \in \operatorname{Syl}_{2}(S)$. Then $\mathbf{N}_{S}(Q)=$ $Q \times C_{(q-\epsilon))_{2} / 3}$.

(ii) If $L=E_{6}^{\epsilon}(q)_{\mathrm{sc}}$ and $Q \in \operatorname{Syl}_{2}(L)$, then $\mathbf{N}_{L}(Q)=Q \times C$ where $C$ is abelian of order $(q-\epsilon)_{2^{\prime}}$.

(iii) $H=E_{6}^{\epsilon}(q)_{\text {ad }}$ has exactly $8(q-\epsilon-3)$ irreducible characters of odd degree that lie above non-unipotent characters of $S=[H, H]$. These characters belong to $q-\epsilon-3$ rational Lusztig series $\mathcal{E}(H,(s)), 8$ in each series, and $\left|\mathbf{C}_{H *}(s)\right|=$ $(q-\epsilon)\left|\operatorname{Spin}_{10}^{\epsilon}(q)\right|$. Each of these characters is irreducible over $S$.

Proof. (i) This is [KM, Theorem 6(c)].

(ii) We can identify $S$ with $L / Z$ for $Z:=\mathbf{Z}(L) \cong C_{3}$. Now (i) implies that $\mathbf{N}_{L}(Q)=Q \times C$, where $C / Z \cong C_{(q-\epsilon)_{2^{\prime}} / 3}$ and so $C$ is abelian of order $(q-\epsilon)_{2^{\prime}}$.

(iii) According to $[\mathrm{Lu}], H$ has $8(q-\epsilon)$ irreducible characters of odd degree. Among them, 24 restrict irreducibly to 8 unipotent characters of $S$, so the remaining $8(q-$ $\epsilon-3)$ all lie above non-unipotent characters of $S$. We identify $H^{*}$ with $L$. Now if $s$ is the label of any such series, then $\left[L: \mathbf{C}_{L}(s)\right]$ is odd, and so we may assume that $s \in \mathbf{C}_{L}(Q)=\mathbf{Z}(Q) \times C=Z\left(\mathbf{N}_{L}(Q)\right)$ for $Q \in \operatorname{Syl}_{2}(L)$ by (ii).

On the other hand, by [FJ], $L$ has $q-\epsilon-3$ conjugacy classes of semisimple elements $t \in L$ with centralizer $\mathcal{C}$ in the underlying algebraic group $\mathcal{G}$ of type $D_{5} T_{1}$ (i.e. $\mathcal{C}$ is a connected reductive group where $\mathbf{Z}(\mathcal{C})^{\circ}$ is a one-dimensional torus and $[\mathcal{C}, \mathcal{C}]$ is of type $\left.D_{5}\right)$, and $\left|\mathbf{C}_{L}(t)\right|=(q-\epsilon)\left|\operatorname{Spin}_{10}^{\epsilon}(q)\right|$; in particular, $2 \nmid\left[L: \mathbf{C}_{L}(t)\right]$.

Next, we note that a finite Lie-type group $Y$ of type $D_{5}^{\alpha}$ over $\mathbb{F}_{q}$ has 8 unipotent characters of odd degree, and these degrees are:

$1, q^{20}, q \Phi_{5} \Phi_{6}, q^{13} \Phi_{5} \Phi_{6}, q^{3} \Phi_{3} \Phi_{5} \Phi_{8} / 2, q^{7} \Phi_{3} \Phi_{5} \Phi_{8} / 2, q^{3} \Phi_{5} \Phi_{6} \Phi_{8} / 2, q^{7} \Phi_{5} \Phi_{6} \Phi_{8} / 2$ if $\alpha=+$, and

$1, q^{20}, q \Phi_{3} \Phi_{10}, q^{13} \Phi_{3} \Phi_{10}, q^{3} \Phi_{3} \Phi_{8} \Phi_{10} / 2, q^{7} \Phi_{3} \Phi_{8} \Phi_{10} / 2, q^{3} \Phi_{6} \Phi_{8} \Phi_{10} / 2, q^{7} \Phi_{6} \Phi_{8} \Phi_{10} / 2$ if $\alpha=+$. (This can be checked directly. Another way to see it is that we can choose $\alpha= \pm$ such that $q \equiv \alpha(\bmod 4)$. There is no loss to work with $Y=S O_{10}^{\alpha}(q)$, and then, a Sylow $\Phi_{e}$-torus in $Y$, as defined in [M1, $\left.\S 5\right]$, is maximal and isomorphic to $C_{q-\alpha}^{5}$, with the relative Weyl group isomorphic to the Weyl group $C_{2}^{4} \rtimes \mathrm{S}_{5}$ of type $D_{5}$. The latter has 8 irreducible characters of odd degree $(1,1,5,5,5,5,15,15)$. It follows by [M1, Corollary 6.6] that $Y$ has 8 unipotent characters of odd degree. As $E_{6}^{\alpha}(q)$ ad has exactly $8(q-\alpha-\operatorname{gcd}(3, q-\alpha))$ odd-degree characters lying above non-unipotent characters of $S$, this implies that these 8 degrees are as listed above. Replacing $q$ by $-q$, we see that $S O_{10}^{-\alpha}(q)$ has at least 8 , hence exactly 8 (by counting in $\left.\operatorname{Irr}_{2^{\prime}}\left(E_{6}^{-\alpha}(q)_{\text {ad }}\right)\right)$, odd-degree unipotent characters.)

It remains to show that each of the above $8(q-\epsilon-3)$ characters is irreducible over $S$. Let $\chi \in \mathcal{E}(H,(s))$ be such a character. Consider any $z \in Z$ and the corresponding character $\lambda_{z} \in \operatorname{Irr}(H / S)$. Then $\lambda_{z} \chi \in \mathcal{E}(H,(s z))$. As mentioned above, $s$ and $s z$ 
both belong to $\mathbf{C}_{L}(Q)=Z\left(\mathbf{N}_{L}(Q)\right)$. By Lemma 4.12 applied to $(G, K)=(L, L), s$ and $s z$ can be $L$-conjugate only when $z=1$. Thus

$$
|\{\lambda \in \operatorname{Irr}(H / S) \mid \lambda \chi=\chi\}|=1
$$

and so $\chi_{S}$ is irreducible by $[\mathrm{KT}$, Lemma 3.2(i)].

Proposition 4.14. Suppose we are in case (ii) of Lemma 4.1. Then $\theta$ extends to $R$.

Proof. (i) Again, we have by [GLS, Theorem 2.5.12] that $A=\operatorname{Aut}(S)=H \rtimes D$, where $H=E_{6}^{\epsilon}(q)_{\mathrm{ad}}, D=\langle\sigma, \tau\rangle$ is abelian of order $2 f, \sigma$ is the field automorphism coming from the map $x \mapsto x^{p}$ of $\overline{\mathbb{F}}_{p}$, and $\tau$ is an involutory graph automorphism considered in [MT, Lemma 4.6]. By Proposition 4.2 we may assume that $p>2$. Now by Lemma 4.13 (iii) we have $I=I_{A}(\theta) \geq H$. We may assume that $R \leq \tilde{R}$, where $\tilde{R} / S \in \operatorname{Syl}_{r}(A / S)$ and $\tilde{R}$ contains the unique Sylow $r$-subgroup $D_{1}$ of $D$. Next, we can write $H X=H \rtimes D_{3}$, where $D_{3}$ is a 2-subgroup of $D_{2}$, the unique Sylow 2subgroup of $D$. Then we choose $\tilde{P} \in \operatorname{Syl}_{2}(A)$ that contains $D_{2}$, and may assume that $P=\tilde{P} \cap I \in \operatorname{Syl}_{2}(X)$ and $Q=\tilde{P} \cap S \in \operatorname{Syl}_{2}(S)$. In particular, $Q$ is $D_{2}$-invariant, and $P=Q \rtimes D_{3}$ as $|H / S|=3$. We again assume by [CS1, Theorem 3.1] that the Jordan decomposition of $H$ is $D$-equivariant.

(ii) Suppose that $\theta$ is $\tau$-invariant. As $H \triangleleft A, \tau$ acts on the set $\operatorname{Irr}(H \mid \theta)$, which consists of $|H / S|=3$ characters by Lemma 4.13(iii). Since $|\tau|=2$, $\tau$ fixes some $\chi \in \operatorname{Irr}(H \mid \theta)$. On the other hand, if $\chi \in \mathcal{E}(H,(s))$, then the $D$-equivariance and Lemma 4.6 of $[\mathrm{MT}]$ show that $\chi^{\tau} \in \mathcal{E}(H,(\tau(s)))=\mathcal{E}\left(H,\left(s^{-1}\right)\right)$. It follows that $s$ and $s^{-1}$ are $L$-conjugate. Hence, as shown in the proof of [NT1, Lemma 9.1], the complex conjugation sends $\mathcal{E}(H,(s))$ to $\mathcal{E}\left(H,\left(s^{-1}\right)\right)=\mathcal{E}(H,(s))$. Thus $\bar{\chi}$ is another character of odd-degree in $\mathcal{E}(H,(s))$ of degree equal to $\chi(1)$. As shown in the proof of Lemma 4.13, $\operatorname{Irr}_{2^{\prime}}(H) \cap \mathcal{E}(H,(s))$ consists of 8 characters of pairwise distinct degrees. It follows that $\bar{\chi}=\chi$ and so $\theta$ is real, whence we are done.

(iii) From now on we may assume that $I \not \supset \tau$. Note that $q \geq 5^{3}$ and $3 \mid(q-\epsilon)$ by Lemma 4.1(ii). Now if $H={ }^{2} E_{6}(q)_{\text {ad }}$, then $3 \mid(q+1)$ implies that $f$ is odd, and so $\tau$ is the unique involution in $A / H \cong D$. As $I \geq H,|H / S|=3$, and $X / S \in \operatorname{Syl}_{2}(I / S)$, we conclude that $X=S$ and $P=Q$. But then $\left|\mathbf{N}_{S}(Q) / Q\right|=(q+1)_{2^{\prime}} / 3>1$ by Lemma 4.13(i), a contradiction.

So $H=E_{6}(q)_{\text {ad }}$. Write $f=2^{a} f_{0}$ with $2 \nmid f_{0}$ and let $\sigma_{0}=\sigma^{f_{0}}, q_{0}=p^{f_{0}}$. Now we show that $\sigma_{0}$ and $\tau$ act on $\mathbf{Z}\left(\mathbf{N}_{S}(Q)\right)$ via

$$
\sigma_{1}(t)=t^{q_{0}}, \tau(t)=t^{-1}
$$

for some generator $\sigma_{1}$ of $\left\langle\sigma_{0}\right\rangle$. First, by considering irreducible representations of $S$ over $\overline{\mathbb{F}}_{p}$, and recalling that $t$ is semisimple, we see that $\sigma_{0}(t)$ and $t^{q_{0}}$ are $S$-conjugate, i.e. $t^{q_{0}}$ and $t$ are conjugate $G:=S \rtimes\left\langle\sigma_{0}\right\rangle: t^{q_{0}}=t^{g}$ with $g=h \sigma_{0}$ for some $h \in S$. Applying Lemma 4.12 with $K=S$ and $E=\left\langle\sigma_{0}\right\rangle$ (recall that $Q$ is normalized by 
$\left.D_{2} \geq E\right)$, we see that $t^{q_{0}}=\sigma_{1}(t)$ for some $\sigma_{1} \in\left\langle\sigma_{0}\right\rangle$, and

$$
\left\langle S, \sigma_{1}\right\rangle=\left\langle S, h \sigma_{0}\right\rangle=S E=G,
$$

i.e. $\left\langle\sigma_{1}\right\rangle=E$. For the second relation in (4.13), note that $\tau(t)$ and $t^{-1}$ are $S$ conjugate by [MT, Lemma 4.6]. So we can apply Lemma 4.12 with $K=S, E=\langle\tau\rangle$, and $G=S E$.

Recall that $P=Q D_{3}$ with $D_{3} \leq D_{2}=\left\langle\sigma_{1}, \tau\right\rangle$ and $D_{3} \not \supset \tau$. Hence we can write $D_{3}=\left\langle\sigma_{1}^{2^{b}} \tau^{j}\right\rangle$, where $0 \leq b \leq a$ and $j \in\{0,1\}$. Set $q_{2}:=p^{2^{b}} f_{0}=q_{0}^{2^{b}}$ and $\kappa=(-1)^{j}$. Note that if $j=1$ then $b<a$ as $D_{3} \not \ngtr \tau$ and so $\left(q_{2}+1\right) \mid(q-1)$. Certainly, $\left(q_{2}-1\right) \mid(q-1)$, and so $\left(q_{2}-\kappa\right) \mid(q-1)$ in all cases. In particular, the cyclic factor $C_{(q-1)_{2^{\prime}} / 3}$ of $\mathbf{C}_{S}(Q)$ contains a subgroup $B$ of order

$$
\left(q_{2}-\kappa\right)_{2^{\prime}} / \operatorname{gcd}\left(3,\left(q_{2}-\kappa\right)_{2^{\prime}}\right) \geq 7
$$

(as $2<r \mid f_{0}$ and $p \geq 5$ ). Now, for any $t \in B$, by (4.13) we have that

$$
\sigma_{1}^{2^{b}} \tau^{j}(t)=t^{\kappa q_{2}}=t
$$

and so $\mathbf{C}_{\mathbf{N}_{S}(Q) / Q}(P) \neq 1$, a contradiction.

Theorem 3.1 now follows from Lemma 4.1 and Propositions 4.11 and 4.14.

Remark 4.15. (i) Note that Theorem 3.1 does not hold without the assumption that $X$ has a self-normalizing Sylow 2-subgroup. Indeed, let $p>2$ be any prime and let $r$ be a prime such that

$$
p \mid(r-1) .
$$

Set $q=r^{p}$, so that $p(r-1) \mid(q-1)$ and consider $S=P S L_{r}(q)$. Let $\alpha \in \mathbb{F}_{q}^{\rtimes}$ be of order $p(r-1)$ and set

$$
s:=\operatorname{diag}(\underbrace{\alpha, \alpha, \ldots, \alpha}_{p-1}, \alpha^{1-p}) \in L=S L_{p}(q) .
$$

Viewing $L=H^{*}$ for $H=P G L_{p}(q)$, we see that $s$ corresponds to a semisimple character $\chi \in \operatorname{Irr}_{2^{\prime}}(H)$ of odd degree $\left(q^{p}-1\right) /(q-1)$. It is easy to see (for instance by degree consideration) that $\theta:=\chi_{S}$ is irreducible. Next, let $\sigma$ denote the field automorphism of order $p$ of $H$ induced by the map $x \mapsto x^{r}$. Then

$$
\frac{\sigma(\alpha)}{\alpha}=\frac{\sigma\left(\alpha^{1-p}\right)}{\alpha^{1-p}}=\alpha^{r-1}
$$

and so $\sigma(s) / s=t:=\alpha^{r-1} \cdot 1_{V} \in \mathbf{Z}(L)$, where $V=\mathbb{F}_{q}^{p}$ denotes the natural $L$-module. Thus $\chi^{\sigma}=\chi \lambda_{t}$, where $\lambda_{t} \in \operatorname{Irr}(H / S)$. As $\theta=\chi_{S}$, it follows that $\theta$ is $\sigma$-invariant, and so $I:=I_{\operatorname{Aut}(S)}(\theta)$ contains $\langle H, \sigma\rangle$. However, $\theta$ does not extend to $I$. Otherwise, there must be some $\tilde{\chi} \in \operatorname{Irr}(\langle H, \sigma\rangle)$ extending $\theta$, and so $\chi^{*}:=(\tilde{\chi})_{H}$ is $\sigma$-invariant. As $\left(\chi^{*}\right)_{S}=\theta=\chi_{S}$, we have $\chi^{*}=\chi \lambda_{v}$ for some $v=\beta \cdot 1_{V}$, with

$$
\beta^{p}=1 \text {. }
$$


Recall that $\chi \in \mathcal{E}(H,(s))$, so $\chi^{*} \in \mathcal{E}(H,(\beta s))$. The $\sigma$-invariance of $\chi^{*}$ now implies that $\sigma(\beta s)$ and

$$
\beta s=\operatorname{diag}(\underbrace{\alpha \beta, \alpha \beta, \ldots, \alpha \beta}_{p-1}, \alpha^{1-p} \beta)
$$

are $L$-conjugate. It follows that $\alpha \beta=\sigma(\alpha \beta)=(\alpha \beta)^{r}$ and so

$$
\alpha^{r-1}=\beta^{1-r}=1
$$

by (4.14) and (4.15). But this is a contradiction, as we chose $\alpha$ of order $p(r-1)$.

(ii) The example in (i) also shows that extendibility results, eg. [CS2, Theorem 4.1], do not imply Theorem 3.1.

\section{A consequence of the McKay-Galois conjecture}

A consequence of the Galois version of the McKay conjecture is that the character table of a finite group $G$ determines if $G$ has self-normalizing Sylow $p$-subgroups [N2]. While for $p$ odd this claim is now a theorem [NTT], the case $p=2$ remains open. Specifically, it is expected that a finite group $G$ has a self-normalizing Sylow 2 -subgroup if and only if every irreducible odd-degree complex character of $G$ is $\sigma$-fixed, where $\sigma$ is the Galois automorphism defined in $\S 2$. A reduction to simple groups of this statement has been carried out in [SF]. Using Theorem 3.3, we can offer a shorter reduction of one of the implications.

Theorem 5.1. Let $G$ be a finite group with a self-normalizing Sylow 2-subgroup. Assume that, whenever $H$ is an almost simple group involved in $G$ with a selfnormalizing Sylow 2-subgroup $Q$ and $H=F^{*}(H) Q$, every $\gamma \in \operatorname{Irr}_{2^{\prime}}(H)$ is $\sigma$-fixed. Then every $\chi \in \operatorname{Irr}_{2^{\prime}}(G)$ is $\sigma$-fixed.

Proof. We argue by induction on $|G|$. Set $p=2$ and let $P \in \operatorname{Syl}_{p}(G)$. Since $\mathbf{N}_{G}(P)=$ $P$, notice that we have that $G / G^{\prime}$ is a 2-group, by the Frattini argument.

Let $\chi \in \operatorname{Irr}_{p^{\prime}}(G)$. Let $N$ be a minimal normal subgroup of $G$. Let $\theta \in \operatorname{Irr}(N)$ be $P$-invariant and lying under $\chi$, and let $\psi \in \operatorname{Irr}(T)$ be the Clifford correspondent of $\chi$ over $\theta$. If $T<G$, by induction we have that $\psi^{\sigma}=\psi$, and therefore $\chi=\psi^{G}$ is also fixed by $\sigma$. So we may assume that $T=G$. By Theorem 3.3, we have that $\theta$ extends to $G$. Let $\rho \in \operatorname{Irr}(G)$ be an extension of $\theta$. By Gallagher's theorem, we have that $\chi=\rho \tau$ for some $\tau \in \operatorname{Irr}_{p^{\prime}}(G / N)$. By induction, $\tau$ is $\sigma$-fixed, so it suffices to show that we can choose $\rho$ to be $\sigma$-fixed.

If $N$ is a $p^{\prime}$-group, then $N \leq \operatorname{Ker}(\chi)$ by the Glauberman correspondence, and we are done by induction. Suppose that $N$ is a $p$-group. Then $\theta$ is linear and $\rho$ is linear. Then $\rho$ has 2-power order and therefore is $\sigma$-fixed.

Hence, we may assume that $N$ is a direct product of isomorphic non-abelian simple groups. Suppose that $N P<G$. Then $\rho_{N P}$ is $\sigma$-fixed by induction. In particular, 
$\theta=\left(\rho_{N P}\right)_{N}$ is $\sigma$-fixed. We now have that $\rho$ and $\rho^{\sigma}$ are two extensions of $\theta$, and therefore

$$
\rho^{\sigma}=\lambda \rho
$$

for some $\lambda \in \operatorname{Irr}(G)$ linear with 2-power order. Now,

$$
\rho_{N P}=\left(\rho_{N P}\right)^{\sigma}=\left(\rho^{\sigma}\right)_{N P}=\lambda_{N P} \rho_{N P}
$$

and we conclude by Gallagher's theorem that $\lambda_{N P}=1_{N P}$. Then $\lambda_{P}=1_{P}$ and therefore $\lambda=1_{G}$ (as $G / G^{\prime}$ is a 2-group). Thus $\rho$ is $\sigma$-fixed and we are done in this case too. Therefore, we may assume that $G=N P$, and that $N$ is the only minimal normal subgroup of $G$. We have that $N=S^{x_{1}} \times \cdots \times S^{x_{t}}$ for some non-abelian simple group $S$. If $H=\mathbf{N}_{G}(S)$ and $Q=P \cap H$, then we know that $Q$ is selfnormalizing in $Q S$ by Lemma 3.2. Write $\theta=\theta_{1} \times \cdots \times \theta_{t}$, where $\theta_{i} \in \operatorname{Irr}_{p^{\prime}}\left(S^{x_{i}}\right)$. Since $\theta$ is $P$-invariant, we have that $\theta_{1}$ is $Q$-invariant. In particular $\theta_{1}$ extends to some $\gamma \in \operatorname{Irr}(S Q)$. If $t>1$, then by induction we have that $\gamma$ is $\sigma$-invariant, and therefore $\theta_{1}=\gamma_{S}$ is $\sigma$-invariant. Since $\theta_{i}=\left(\theta_{1}\right)^{x_{i}}$, we deduce that $\theta_{i}$ and thus $\theta$ are

$\sigma$-invariant. Now, $\theta$ has a canonical extension to $G$ by [I2, Corollary (6.28)], which is necessarily $\sigma$-invariant, and so we are done again. This leave us with the case $t=1$, which is true by hypothesis.

Added in proof. While the paper was in submission, we learned that the McKay conjecture for $p=2$ was proved by G. Malle and B. Späth [MS].

\section{REFERENCES}

[CS1] M. Cabanes and B. Späth, Equivariance and extendibility in finite reductive groups with connected center, Math. Z. 275 (2013), 689-713.

[CS2] M. Cabanes and B. Späth, Equivariant character correspondences and inductive McKay condition for type A, J. reine angew. Math. (to appear).

[C] R. Carter, 'Finite Groups of Lie type: Conjugacy Classes and Complex Characters', Wiley, Chichester, 1985.

[FJ] P. Fleischmann and I. Janiszczak, The semisimple conjugacy classes of finite groups of Lie type $E_{6}$ and $E_{7}$, Comm. Algebra 21 (1993), 93-61.

[GLS] D. Gorenstein, R. Lyons, and R. Solomon. The Classification of the Finite Simple Groups, Number 3. Part I. Chapter A, volume 40 of Mathematical Surveys and Monographs. American Mathematical Society, Providence, RI, 1998.

[I1] I. M. Isaacs, Character correspondences in solvable groups, Adv. in Math. 43 (1982), 284306.

[I2] I. M. Isaacs, 'Character Theory of Finite Groups', AMS-Chelsea, Providence, 2006.

[IMN] I. M. Isaacs, G. Malle, and G. Navarro, A reduction theorem for the McKay conjecture, Invent. Math. 170 (2007), 33-101.

$[\mathrm{KT}] \quad$ A. S. Kleshchev and Pham Huu Tiep, Representations of finite special linear groups in non-defining characteristic, Adv. Math. 220 (2009), 478-504.

$[\mathrm{KM}] \quad$ A. S. Kondratiev and V. D. Mazurov, 2-signalizers of finite simple groups, Algebra and Logic 42 (2003), 333-348. 
[Lu] F. Lübeck, Character degrees and their multiplicities for some groups of Lie type of rank $<9$, http://www.math.rwth-aachen.de/ Frank.Luebeck/chev/DegMult/index.html

[L] G. Lusztig, Irreducible representations of finite classical groups, Invent. Math. 43 (1977), $125-175$.

[M1] G. Malle, Height 0 characters of finite groups of Lie type, Represent. Theory 11 (2007), $192-220$.

[M2] G. Malle, Extensions of unipotent characters and the inductive McKay condition, J. Algebra 320 (2008), 2963-2980.

[MS] G. Malle and B. Späth, Characters of odd degree, preprint, arXiv:1506.07690.

$[\mathrm{MT}]$ S. Marinelli and Pham Huu Tiep, Zeros of real irreducible characters of finite groups, Algebra Number Theory 7 (2013), 567-593.

[M] J. McKay, Irreducible representations of odd degree, J. Algebra 20 (1972), 416-418.

[N2] G. Navarro, The McKay conjecture and Galois automorphisms, Annals of Math. 160 (2004), 1129-1140.

[NT1] G. Navarro and Pham Huu Tiep, Rational irreducible characters and rational conjugacy classes in finite groups, Trans. Amer. Math. Soc. 360 (2008), 2443-2465.

[NT2] G. Navarro and Pham Huu Tiep, Characters of relative $p^{\prime}$-degree over normal subgroups, Annals of Math. 178 (2013), 1135-1171.

[NTT] G. Navarro, Pham Huu Tiep, and A. Turull, p-rational characters and self-normalizing Sylow p-subgroups, Represent. Theory 11 (2007), 84-94.

[NTV] G. Navarro, Pham Huu Tiep, and C. Vallejo, McKay natural correspondences on characters, Algebra Number Theory 8 (2014), 1839-1856.

[SF] A. A. Schaeffer Fry, Self-normalizing Sylow subgroups and Galois automorphisms, Comm. Algebra (to appear).

[S1] B. Späth, Sylow $d$-tori of classical groups and the McKay conjecture. I, J. Algebra 323 (2010), 2469-2493.

[S2] B. Späth, Sylow $d$-tori of classical groups and the McKay conjecture. II, J. Algebra 323 (2010), 2494-2509.

[S3] B. Späth, A reduction theorem for the Alperin-McKay conjecture, J. reine angew. Math. 680 (2013), 153-189.

[TZ] Pham Huu Tiep and A. E. Zalesski, Real conjugacy classes in algebraic groups and finite groups of Lie type, J. Group Theory 8 (2005), 291-315.

Departament d’Àlgebra, Universitat de València, 46100 Burjassot, ValÈncia, Spain

E-mail address: gabriel.navarro@uv.es

Department of Mathematics, University of Arizona, Tucson, AZ 85721, USA

E-mail address: tiep@math.arizona.edu 